Pure and Applied Mathematics Quarterly

Volume 5, Number 4

(Special Issue: In honor of

John Tate, Part 1 of 2)

$1385-1427,2009$

\title{
From Tate's Elliptic Curve to Abeloid Varieties
}

\author{
W. Lütkebohmert \\ Dedicated to John Tate.
}

\begin{abstract}
In this article we will study smooth rigid analytic group objects over local fields whose underlying variety is proper and connected. Starting in dimension 1 where such an object is automatically algebraic and projective, we are concerned with elliptic curves. Up to finite base field extension, an elliptic curve has good reduction or multiplicative reduction. To understand the geometric meaning of the multiplicative case was the incentive for J. Tate to introduce rigid analytic theory. Moreover it turned out that the degeneracy of abelian varieties over local fields could also be explained by rigid geometry. In higher dimension the situation is much more complicated than in dimension 1. Namely, there exist non-algebraic objects, the so-called abeloid varieties. In this article we will revisit the main steps in building the theory of rigid geometry and we will describe the main ideas for elaborating the structure of abeloid varieties and how to classify the abelian varieties among them.
\end{abstract}

Keywords: Formal and Rigid Geometry, Abelian varieties, Abeloid varieties, Picardfunctor, Artin Approximation

\section{TAte's Elliptic CURVE}

The invention of rigid analysis was closely related to uniformization problems in algebraic geometry and it starts with a pioneering piece of work of Tate [39], dating back to 1959, which was unpublished for a long time until 1995. Over a

Received November 19, 2006. 
completely valued non-archimedean field, Tate constructed elliptic curves by considering "analytic" quotients $\mathbb{G}_{m, K} / q^{\mathbb{Z}}$ where $q \in K^{\times}$has absolute value $|q|<1$. Tate's uniformization of elliptic curves with non-integral $j$-invariant was elaborated by Roquette [37]. A certain generalization of Tate's construction of elliptic curves to the case of several dimensions was given by Morikawa [29]. Then in the early 1970's, it was generalized in two ways, namely to curves of higher genus having split degenerate reduction by Mumford [30] and to abelian varieties of arbitrary dimensions by Mumford [31] and Raynaud [34]. Mumford worked over complete noetherian rings and constructed families of polarized abelian varieties. On the other hand, Raynaud studied the uniformization of abelian varieties over local fields. Neither considered the geometric case of an algebraically closed nonarchimedean valued field. In the latter case, Mumford's curves were thoroughly investigated from the rigid analytic point of view by Gerritzen [13] and, the same was done for abelian varieties by Bosch and the author in [4].

Tate's elliptic curve was an important tool for Deligne and Rapoport for studying the points of infinity of the arithmetic moduli scheme of elliptic curves [9]. Later Faltings and Chai used the work of Mumford and Raynaud in constructing the toroidal compactification of the arithmetic moduli scheme of principally polarized abelian varieties [12].

The uniformization of elliptic curves can be understood in terms of function theory on simple domains like discs and annuli. However a satisfying treatment of curves of higher genus or abelian varieties needs a more sophisticated theory of analytic functions which allows the notion of global analytic spaces. This was the motivation of Tate to work out the details in a seminar at Harvard 1961 where he started rigid geometry. These notes were later published in Inventiones in 1970; cf. [38]. By introducing an extra topological structure, Tate overcame the problem of analytic continuation over totally disconnected ground fields; thus making the impossible possible, as Remmert named it. Thereby he layed the foundation of modern rigid geometry. The theory was later worked out by the school of Grauert and Remmert resp. by Kiehl; for details see the monography [3]. While Mumford was working exclusively in the framework of formal algebraic geometry, Raynaud presented a program [36] which explained the connection between formal and rigid geometry. Thereby it helps to understand the geometry behind the constructions of Mumford. Details of Raynaud's program were worked out by Bosch and the author in $[6]$. 
For stating the results we fix the notations for this article. Let $R$ be a complete discrete valuation ring with uniformizing parameter $\pi$, residue field $k$ and field of fractions $K$. Most of the results are true for non-archimedean fields which are complete with respect to an arbitrary valuation of rank 1 ; in that case $\pi$ is an element of $K^{\times}$with $0<|\pi|<1$ and $R$ consists of all elements $c \in K$ with $|c| \leq 1$. The latter are referred to as local fields.

The following statements can be found in $[37, \S 3$, VI \& VII].

Theorem 1.1 (Tate). Let $K$ be a local field of arbitrary characteristic as above and let $q \in K^{\times}$with $0<|q|<1$. Then the field of meromorphic q-periodic functions on $\mathbb{G}_{m, K}$ is an elliptic function field $F(q)$; i.e. finitely generated of transcendence degree 1 over $K$. More precisely $F(q)=K(\wp, \tilde{\wp})$ where

$$
\begin{aligned}
& \wp(\xi)=\sum_{n \in \mathbb{Z}} \frac{q^{n} \xi}{\left(1-q^{n} \xi\right)^{2}}-2 \cdot s_{1} \\
& \tilde{\wp}(\xi)=\sum_{n \in \mathbb{Z}} \frac{q^{2 n} \xi^{2}}{\left(1-q^{n} \xi\right)^{3}}+s_{1}
\end{aligned}
$$

where

$$
s_{\ell}:=\sum_{m \geq 1} \frac{m^{\ell} q^{m}}{1-q^{m}} \text { for } \ell \in \mathbb{N} .
$$

The associated projective curve $E(q)$ is given by the inhomogeneous equation

$$
\tilde{\wp}^{2}+\wp \cdot \tilde{\wp}=\wp^{3}+B \cdot \wp+C
$$

for $B:=-5 \cdot s_{3}, C:=\frac{1}{12}\left(5 \cdot s_{3}+7 \cdot s_{5}\right)$ which are in $q \mathbb{Z}[[q]]$. Its $j$-invariant is

$$
j(q)=\frac{(1-48 \cdot B)^{3}}{\Delta}=\frac{1}{q}+R(q)
$$

where

$$
\begin{aligned}
& R(q)=744+196884 \cdot q+\ldots \in \mathbb{Z}[[q]] \\
& \Delta(q)=B^{2}-C-64 \cdot B^{3}+72 \cdot B C-432 \cdot C^{2}=q \cdot \prod_{n \geq 1}\left(1-q^{n}\right)^{24} \in \mathbb{Z}[[q]]
\end{aligned}
$$

To every element $j \in K$ with $|j|>1$ there exists one and only one $q \in K$ with $0<|q|<1$ such that $j=j(q)$.

For the proof Tate had to introduce analytic functions on $\mathbb{G}_{m, K}$. This is done by postulating the Laurent expansion principle. This is exactly the point where $p$-adic analysis enters the story. For introducing $q$-periodic meromorphic 
functions on $\mathbb{G}_{m, K}$, one had to handle analytic functions only on simple domains like discs or annuli. But it is not obvious how to introduce an analytic structure on the projective curve $E(q)$ associated to $F(q)$ and how to interpret the function given by $\wp$ and $\tilde{\wp}$ as an analytic morphism from $\mathbb{G}_{m, K} / q^{\mathbb{Z}}$ to $E(q)$. A satisfying treatment would require a theory of analytic functions which includes the notion of Riemann surfaces. Although one could feel the geometry behind it, to explain it requires rigid analytic geometry. Later, Raynaud gave a geometric construction of the general Tate curve over the power series ring $\mathbb{Z}[[q]]$ in terms of formal schemes; cf. [9, Sect. VII].

Theorem 1.2 (Tate). Let $E$ be an elliptic curve over a local field $K$. After finite extension of the ground field there are two possibilities:

(i) If $|j(E)| \leq 1$ then $E$ has good reduction.

(ii) If $|j(E)|>1$ then $E$ is isomorphic to an analytic torus $\mathbb{G}_{m, K} / q^{\mathbb{Z}}$ for a unique $q \in K^{\times}$with $0<|q|<1$. The $j$-invariant bijectively depends on $q$ by a series

$$
j(q)=\frac{1}{q}+f(q)
$$

with $f(q) \in R[[q]]$. Thus $j(q)$ is converging on the open punctured disc and $j$ gives rise to a biholomorphic map

$$
j:\left\{q \in K^{\times} ; 0<|q|<1\right\} \longrightarrow\left\{j \in K^{\times} ;|j|>1\right\} .
$$

Over an algebraically closed field, an elliptic curve is uniquely determined by its $j$-invariant. If the characteristic of the ground field is $\neq 2$, any elliptic curve $E$ is given by a Legendre equation in the projective plane

$$
E \cong E(\lambda):=V\left(Y^{2} \cdot Z-X \cdot(X-Z) \cdot(X-\lambda Z)\right) \subset \mathbb{P}_{K}^{2}
$$

with $\lambda \in K-\{0,1\}$. Its $j$-invariant is

$$
j(E)=2^{8} \frac{\left(1-\lambda+\lambda^{2}\right)^{3}}{\lambda^{2}(1-\lambda)^{2}}
$$

which is invariant under the substitution $\lambda \longmapsto(1-\lambda)$ reflecting the isomorphism $E(\lambda) \cong E(1-\lambda)$. Over a valued field $K$, for $E(\lambda)$ with $\lambda \in K$ we may assume $|\lambda| \leq 1$. Then

$E(\lambda)$ has good reduction if and only if $|\lambda|=1$ and $|1-\lambda|=1$.

$E(\lambda)$ has multiplicative reduction if and only if $0<|\lambda|<1$ or $|1-\lambda|<1$. 
Good reduction means that the polynomial $P(X):=X \cdot(X-1) \cdot(X-\lambda)$ has three distinct roots in the reduction, whereas multiplicative reduction means that two roots of $P(X)$ collapse in the reduction.

The relationship of the modulus $q$ with the Legendre representation $E(\lambda)$ for $0<|\lambda|<1$ is the following

$$
q(\lambda)=c_{2} \lambda^{2}+c_{3} \lambda^{3}+\ldots
$$

with $\left|c_{2}\right|=1$ and $\left|c_{i}\right| \leq 1$ for $i \in \mathbb{N}$. In particular, there are always exactly two values $\lambda_{1}, \lambda_{2}$ such that $\left|\lambda_{i}\right|<1$ with $j\left(\lambda_{1}\right)=j\left(\lambda_{2}\right)$; cf. $[3, \S 9.7]$.

\section{Formal AND RIGID GEOMETRY}

The building blocks of $p$-adic analysis over a local field $K$ are the Tate algebras

$$
T_{n}:=\left\{\sum_{i=0}^{\infty} a_{i} \xi^{i} \in K\left\langle\xi_{1}, \ldots, \xi_{n}\right\rangle ; \lim _{i \rightarrow \infty} a_{i}=0\right\}
$$

and its residue algebras; the so-called affinoid algebras

$$
A_{K}:=T_{n} / \mathfrak{a}
$$

The associated geometric objects are the affinoid spaces $\operatorname{Sp}\left(A_{K}\right)$ defined as the set of maximal ideals of $A_{K}$; for example $\operatorname{Sp}\left(T_{n}\right)$ is just the set of points in the affine $n$-space $\mathbb{A}_{K}^{n}$ where the coordinates $\xi_{i}$ take absolute values $\leq 1$. In order to build a useful $p$-adic theory, one has to ask at least two principles; namely the analytic continuation and the global expansion of analytic functions on discs. This poses a serious problem because the natural topology given by the valuation of $K$ is totally disconnected. Therefore one has to provide $p$-adic analytic spaces with an extra topology which forces a non-trivial notion of connectedness. The definition of rigid spaces [38] was rather clumsy at the beginning. Substantial simplifications are due to Gerritzen and Grauert by introducing the notion of rational domains; cf. [14]. As a result, admissible open sets and coverings of rigid spaces became easier to handle.

Definition 2.1. Let $X=\operatorname{Sp}\left(A_{K}\right)$ be an affinoid space and let $f_{0}, \ldots, f_{r} \in A_{K}$ without common zeros. Then

$$
X_{j}:=X\left(f_{0} / f_{j}, \ldots, f_{r} / f_{j}\right):=\left\{x \in X ;\left|f_{i}(x)\right| \leq\left|f_{j}(x)\right| \text { for } i=0, \ldots, r\right\}
$$


is called a rational affinoid subdomain of $X$ with structure ring

$$
\mathcal{O}_{X}\left(X_{j}\right):=A_{K}\left\langle\zeta_{0} / \zeta_{j}, \ldots, \zeta_{r} / \zeta_{j}\right\rangle /\left(\zeta_{i} f_{j}-\zeta_{j} f_{i} ; i=0, \ldots, r\right) .
$$

$\left(X_{0}, \ldots, X_{r}\right)$ is called a rational covering of $X$.

The structure sheaf $\mathcal{O}_{X}$ is acyclic for rational coverings.

At the same time Kiehl simplified Tate's theory as well by using Grothendieck topologies and obtained some fundamental results on coherent modules; the rigid analytic analogues to Grauert's Direct Image Theorem and of Theorem A and B of Cartan and Serre; cf. [23] and [24]. Thus Serre's GAGA could be carried over to rigid geometry verbatim. In 1974 Raynaud proposed a program [36] shedding new light into the theory by interpreting rigid geometry as the generic fiber of formal schemes over the formal spectrum $\operatorname{Spf}(R)$ of a complete discrete valuation ring. This was first elaborated by Mehlmann in [27]. A more detailed treatment is [6, Part $1 \&$ Part 2].

Instead of starting directly with affinoid algebras, Raynaud started with $R$ algebras of type

$$
A:=R\left\langle\xi_{1}, \ldots,, \xi_{n}\right\rangle / \mathfrak{a}
$$

without $R$-torsion and of topologically finite presentation; called admissible $R$ algebras. The formal spectrum $\operatorname{Spf}(A)$ with ideal of definition given by a parameter $\pi$ of $R$ is called an admissible affine formal scheme. Setting

$$
A_{\text {rig }}:=A \otimes_{R} K=K\left\langle\xi_{1}, \ldots, \xi_{n}\right\rangle / \mathfrak{a} \cdot K,
$$

the maximal spectrum $X_{\text {rig }}:=\operatorname{Sp}\left(A_{\text {rig }}\right)$ is called the generic fiber of the formal affine scheme $X:=\operatorname{Spf}(A)$. The crucial idea of Raynaud was the use of formal blowing ups.

Definition 2.2. Let $A$ be an admissible $R$-algebra and let $X=\operatorname{Spf}(A)$ be an affine formal scheme. For an open ideal $\mathcal{I}=\left(f_{0}, \ldots, f_{r}\right) \subset A$; i.e. $\pi^{n} \in \mathcal{I}$ for some $n \in \mathbb{N}$; the formal blowing up $\mathbb{P}_{X}^{r} \supset X^{\prime} \longrightarrow X$ of $\mathcal{I}$ on $\operatorname{Spf}(A)$ is the completion of the algebraic blowing up of $\mathcal{I}$ on $\operatorname{Spec}(A)$ with respect to the special fiber.

It is clear that the generic fiber is not changed since $\mathcal{I} \otimes K=A_{\text {rig }}$, and that the canonical covering of $\mathbb{P}_{X}^{r}$ induces the rational covering on $X^{\prime}$ defined by the chosen system of generators of $\mathcal{I}$. One can generalize this procedure for any admissible formal scheme over $\operatorname{Spf}(R)$; i.e. a formal scheme which is covered by 
open subschemes of type $\operatorname{Spf}(A)$ as above where the ideal of definition is given by a parameter $\pi \in R$. This way one gets a rigid space $X_{\text {rig }}$ associated to any admissible formal scheme over $\operatorname{Sp}(R)$; called the generic fiber of $X$.

Theorem 2.3 (Existence of Integral Models). The functor rig : (Admissible Formal $R$-Schemes $) \longrightarrow$ (Rigid $K$-Spaces $)$

gives rise to an equivalence between

(1) the category of quasi-compact admissible formal R-schemes, localized by admissible formal blowing-ups, and

(2) the category of rigid $K$-spaces which are quasi-compact and quasi-separated.

The main tool for proving such a theorem was provided by the paper [35] of Raynaud and Gruson. In the following we will state some consequences of it to explain the relationship of formal and rigid geometry.

Theorem 2.4 (Flattening). Let $f: X \rightarrow Y$ be a morphism of quasi-compact admissible formal $R$-schemes. Let $n \in \mathbb{N}$ be an integer. If $f_{\text {rig }}$ is flat in dimension $\geq n$, then there exists an admissible formal blowing-up $Y^{\prime} \rightarrow Y$ such that the induced map $f^{\prime}: X^{\prime} \rightarrow Y^{\prime}$ is flat in dimension $\geq n$ where $X^{\prime}$ is the strict transform of $X$ with respect to $Y^{\prime} \rightarrow Y$.

This new insight made the theorems of Kiehl on coherent modules [23] and [24] into a corollary of well known facts in algebraic geometry [25, §1]. Moreover it gives access to the work of Grothendieck [17]. Many interesting geometric results could be shown by this technique; cf. [6, Part 2, $5.10 \& 11]$.

Corollary 2.5. Let $f: X \rightarrow Y$ be a morphism of quasi-compact admissible formal $R$-schemes as above.

(a) If $\operatorname{dim}(X / Y)_{\text {rig }} \leq n$ then there exists an admissible formal blowing-up $Y^{\prime} \rightarrow Y$ such that the induced map $f^{\prime}: X^{\prime} \rightarrow Y^{\prime}$ has relative dimension $\leq n$.

(b) If $f_{\text {rig }}$ is quasi-finite, one can choose $Y^{\prime} \rightarrow Y$ such that $f^{\prime}: X^{\prime} \rightarrow Y^{\prime}$ is quasi-finite as well.

(c) If $f_{\text {rig }}$ is an open immersion (resp. closed), then one can choose $Y^{\prime} \rightarrow Y$ such that $f^{\prime}: X^{\prime} \rightarrow Y^{\prime}$ is an open immersion (resp. closed).

(d) If $f_{\text {rig }}$ is flat, then the image of $f_{\text {rig }}$ is a finite union of open affinoid subvarieties of $Y_{\text {rig. }}$. 
An important topic is the finiteness result of Grauert and Remmert solving a question of Tate [38], stating that, for a reduced affinoid $K$-algebra $A_{K}$ over an algebraically closed field $K$, the $R$-algebra $A \subset A_{K}$ consisting of all functions $f \in A_{K}$ with sup-norm $|f| \leq 1$ is of topologically finite type over $R$. A relative version of this result was proved in [6, Part IV, 2.1]. This is in some way the first step towards a statement on relative potential reduction which will be treated in $\S 7$.

Theorem 2.6 (Reduced Fiber Theorem). Let $S$ be an arbitrary admissible formal base scheme. Let $X$ be an admissible formal $S$-scheme such that $X / S$ is flat and $X_{\text {rig }} / S_{\text {rig }}$ has reduced geometric fibers, equidimensional of dimension $d$. Then there is a commutative diagram of admissible formal $S$-schemes

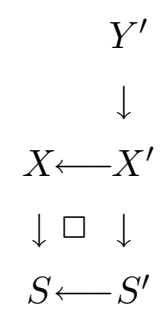

such that

(i) $S^{\prime} \rightarrow S$ is a rig-étale cover, $X^{\prime}=X \times{ }_{S} S^{\prime}$,

(ii) $Y^{\prime} \rightarrow X^{\prime}$ is a finite rig-isomorphism,

(iii) $Y^{\prime} \rightarrow S^{\prime}$ is flat and has reduced geometric fibers.

\section{Mumford's CONSTRUCTION}

From the analytic point of view, at a first glance the generalization of Tate's elliptic curve is the analytic torus

$$
\mathbb{G}_{m, K}^{r} / M_{K}
$$

where a lattice is defined as a closed discrete subgroup $M_{K} \subset \mathbb{G}_{m, K}$ such that

$$
-\log : \mathbb{G}_{m, K}^{r}(\bar{K}) \longrightarrow \mathbb{R}^{r} ;\left(z_{1}, \ldots, z_{r}\right) \longmapsto\left(-\log \left|z_{1}\right|, \ldots,-\log \left|z_{r}\right|\right)
$$

maps $M_{K}(\bar{K})$ to a full lattice in the Euclidean space $\mathbb{R}^{r}$ where $\bar{K}$ is the algebraic closure of $K$. To define such a quotient one needs rigid geometry. As explained in $\S 1$, the latter was not necessary for $r=1$, since the object is algebraic and, hence, there were methods to omit the building of analytic quotients. But for $r \geq 2$ such a quotient does not need to be algebraic. Therefore a more involved 
construction was necessary. In [31] Mumford postulates additional data which imply the existence of an ample line bundle on the quotient and, hence, force the quotient to be algebraic. Somehow he does not form the analytic quotient, his construction automatically produces an abelian variety over $K$ which is the quotient. However the explicit construction of the quotient is hidden. Mumford's construction works even more generally over any noetherian ring $A$ which is complete with respect to an ideal $\mathcal{I}$ of $A$.

Let us start with the most general example of a rigid group variety which is proper and connected. This can illustrate the geometry of such objects before explaining Mumford's construction.

Example 3.1 (Fundamental Example of an Abeloid Variety).

Let $g \geq r \geq 0$ be integers. Let $B \rightarrow \operatorname{Spf}(R)$ be a formal abelian scheme of dimension $(g-r)$; i.e. $B$ is the inductive limit of abelian schemes $B_{n}$ over $\operatorname{Spec}\left(R /\left(\pi^{n+1}\right)\right)$ such that $B_{n-1}=B_{n} \otimes R /\left(\pi^{n}\right)$. Let $E_{1}, \ldots, E_{r}$ be translation invariant $\mathbb{G}_{m}$-torsors over $B_{K}:=B_{\text {rig }}$. Then

$$
\widehat{G}_{K}:=E_{1} \times_{B} \ldots \times_{B} E_{r} \longrightarrow B_{K}
$$

has a commutative group structure due to the Theorem of the Square. Since each $E_{\rho}$ extends to a formal line bundle on $B$, one gets a well-defined absolute value $|\cdot|$ on each $B_{\rho}$. Hence one obtains as before a well defined map

$$
-\log : \widehat{G}_{K}(\bar{K}) \longrightarrow \mathbb{R}^{r} ;\left(z_{1}, \ldots, z_{r}\right) \longmapsto\left(-\log \left|z_{1}\right|, \ldots,-\log \left|z_{r}\right|\right)
$$

Thus one can define a lattice $M_{K} \subset \widehat{G}_{K}$ as before and so one obtains an analytic quotient

$$
G_{K}:=\widehat{G}_{K} / M_{K}
$$

This example depends "versally" on $g^{2}$ modules:

$B$ as deformation of $B_{0}$ depends on $\quad(g-r)^{2} \quad$ modules,

$\widehat{G}_{K}$ as $r$ points in the dual $B^{\prime}$ of $B$ depends on $r \cdot(g-r)$ modules,

$M_{K}$ as $r$ points in $E$ depends on $\quad r \cdot g \quad$ modules.

So the construction of $G_{K}$ is versally in $g^{2}$ modules.

There is a unique largest open subgroup of $G_{K}$; namely

$$
\bar{G}_{K}:=\left\{z=\left(z_{1}, \ldots, z_{r}\right) \in \widehat{G}_{K} ;\left|z_{\rho}\right|=1 \text { for } \rho=1, \ldots, r\right\} .
$$


Definition 3.2. Let $A$ be an excellent normal noetherian ring which is complete with respect to a reduced ideal $\mathcal{I} \subset A$ and let $K:=\operatorname{Frac}(A)$ denote its field of fractions. Set $S:=\operatorname{Spec}(A)$ and $S_{0}=:=\operatorname{Spec}(A / \mathcal{I})$. The data of Mumford's construction in the case of split torus reduction consists of the following:

(1) $\widehat{G}=\mathbb{G}_{m, S}^{r}$ split torus over $S:=\operatorname{Spec}(A)$ of rank $r$

(2) $M \subset \widehat{G}(K)$ a set of periods; i.e. a subgroup isomorphic to $\mathbb{Z}^{r}$

(3) $\lambda: M \rightarrow M^{\prime}:=\operatorname{Hom}_{S}\left(\widehat{G}, \mathbb{G}_{m, S}\right)$ a polarization; i.e. a group homomorphism such that:

(i) $\left\langle m_{1}, \lambda\left(m_{2}\right)\right\rangle=\left\langle m_{2}, \lambda\left(m_{1}\right)\right\rangle$ for all $m_{1}, m_{2} \in M$

(ii) $\langle m, \lambda(m)\rangle \in \mathcal{I}$ for all $m \in M-\{0\}$,

where $\langle\cdot, \cdot\rangle: M \times M^{\prime} \rightarrow \mathbb{G}_{m, K},\left(m, m^{\prime}\right) \mapsto m^{\prime}(m)$, is the canonical pairing.

Theorem 3.3 (Mumford). In the situation of 3.2 there exists the quotient $G:=$ $\widehat{G} / M$ which is a semi-abelian group scheme over $S$ with abelian generic fiber $G_{\eta}:=G \otimes_{S} K$ carrying a polarization $\lambda_{G}$ and special fiber $G \times_{S} S_{0}$ being a split torus.

We have chosen the symbol $\widehat{G}$, since it should be thought of as being the universal covering of the quotient $G_{K}$.

Mumford does not construct the quotient on the generic fiber; instead this is done on the levels of the special fiber over $S_{n}:=\operatorname{Spec}\left(A / \mathcal{I}^{n}\right)$ for all $n \in \mathbb{N}$. As a trick he introduces the notion of a relatively complete model $(\widehat{P}, \widehat{L})$ over $S$. That is a kind of compactification of $\widehat{G}$ by an integral $S$-scheme locally of finite type equipped with an $S$-ample line bundle $\widehat{L}$

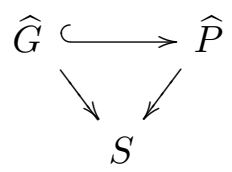

such that the action of $\widehat{G}$ resp. $M$ by translation on $\widehat{G}$ extends to an action on $(\widehat{P}, \widehat{L})$ inducing an $M$-linearization on $\widehat{L}$. The abstract group $M$ acts freely and discontinuously on the special fiber $\widehat{P}_{n}$, and $\widehat{P}$ is covered by the translates of an open $\widehat{G}$-invariant subscheme $U \subset \widehat{P}$ of finite type under $M$. The actions of $\widehat{G}$ and $M$ on $\widehat{L}$ commute up to a twist by $\langle g, \lambda(m)\rangle$ for $g \in \widehat{G}$ and $m \in M$. The completeness is expressed in terms of valuations on the field of rational functions on $\widehat{G}$; namely that certain valuations have centers on $\widehat{P}$. 
Such relatively complete models exist. They are not unique but the ambiguity does not affect the resulting group scheme $G$. A candidate for $\widehat{P}$ is provided by the following construction. Let

$$
\mathcal{R}:=\sum_{n=0}^{\infty} \mathcal{O}_{\widehat{G}}\left(\widehat{G}_{\eta}\right) \cdot \theta^{n}=\sum_{n=0}^{\infty}\left(\bigoplus_{m^{\prime} \in M^{\prime}} K \cdot m^{\prime}\right) \cdot \theta^{n}
$$

be the large graded ring where $\theta$ is an indeterminant. On $\mathcal{R}$ one can easily define the actions of $\widehat{G}$ and $M$ via

$$
\begin{aligned}
\tau_{g}^{*} m^{\prime} & =\left\langle g, m^{\prime}\right\rangle \cdot m^{\prime} \quad \text { and } \tau_{g}^{*} \theta=\theta \\
\tau_{m}^{*} m^{\prime} & =\left\langle m, m^{\prime}\right\rangle \cdot m^{\prime} \quad \text { and } \tau_{m}^{*} \theta=\left\langle m, \lambda_{M}(m)\right\rangle \cdot \lambda_{M}(m) \cdot \theta
\end{aligned}
$$

Inside $\mathcal{R}$ one considers the subring

$$
R_{\Sigma}:=A\left[\left\langle m, \lambda_{M}(m)+\mu\right\rangle \cdot\left(\lambda_{M}(m)+\mu\right) \cdot \theta\right]_{m \in M, \mu \in \Sigma} \subset A[\mu \cdot \theta]_{\mu \in \Sigma}
$$

where $\Sigma \subset M^{\prime}$ is a finite subset containing a basis of $M^{\prime}$ and satisfying $0 \in \Sigma$ and $\Sigma=-\Sigma$. Assume that $\left\langle m, \lambda_{M}(m)+\mu\right\rangle \in A$; otherwise one has to replace $\lambda_{m}$ by a positive multiple. Then

$$
\widehat{P}:=\operatorname{Proj}\left(R_{\Sigma}\right)
$$

is a relatively complete model and, moreover, there is a canonical ample line bundle on it.

Then one proves that $\widehat{P}$ is as close as possible to $\widehat{G}$; namely the generic fibers $\widehat{G}_{\eta}=\widehat{P}_{\eta}$ coincide automatically and the interesting points of $\widehat{G}_{\eta}$ specialize on $\widehat{P}$. Mumford performs the quotient on the $\mathcal{I}$-adic completion $\widehat{\mathfrak{P}}$ of $\widehat{P}$ obtaining a formal scheme $\mathfrak{P}=\widehat{\mathfrak{P}} / M$. This scheme can be algebraized to a scheme $P$ over $S$ which is projective over $S$ due to the presence of the ample line bundle $\widehat{L} / M$. A suitable open subset $G \subset P$ gives the semi-abelian $S$-group scheme one is looking for. It is independent of the choice of $\widehat{P}$; it depends only on $\widehat{G}$ and $M \subset \widehat{G}(K)$. Furthermore $G_{\eta}$ is abelian and $G_{0} \cong \widehat{G}_{0} \cong \mathbb{G}_{m}^{r} \times_{S} S_{0}$. Actually it works as it is illustrated in the following diagram

$$
\begin{gathered}
\widehat{G} \subset \quad \widehat{P}<\frac{\mathcal{I} \text {-adic }}{\text { completion }}-\widehat{\mathfrak{P}} \\
G \subset P<\frac{\mathcal{I} \text {-adic }}{\text { completion }}-\mathfrak{V}
\end{gathered}
$$


We do not see the quotient on the generic fiber; this is hidden in the algebraization process which works due to the presence of the polarization. Without the polarization this method wouldn't work.

Chai and Faltings generalized this construction to the mixed case where $\widehat{G}$ is an extension of an abelian $S$-scheme of positive dimension by a torus $\mathbb{G}_{m, S}^{r}$ [12, Chap. III]. Their construction is very similar to that of Mumford which essentially relies upon the algebraization theorem of Grothendieck. They also need a polarization; however in their case the data for a polarization is much more involved.

Definition 3.4. Let $A$ be an excellent normal noetherian ring which is complete with respect to a reduced ideal $\mathcal{I} \subset A$ and let $K:=\operatorname{Frac}(A)$ be its field of fractions. Set $S:=\operatorname{Spec}(A)$ and $S_{0}:=\operatorname{Spec}(A / \mathcal{I})$. The data of Mumford's construction in the mixed case consists of the following:

(1) $\widehat{G}$ is an extension of an abelian $S$-scheme $B$ by $T:=\mathbb{G}_{m, S}^{r}$.

Such an extension is equivalent to a group homomorphism

$$
\Phi^{\prime}: M^{\prime}:=\operatorname{Hom}_{S}\left(T, \mathbb{G}_{m, S}\right) \longrightarrow B^{\prime}:=\text { dual of } B .
$$

Let $q: \widehat{G} \rightarrow B$ be the map from $\widehat{G}$ to $B$.

(2) $M \subset \widehat{G}(K)$ a set of periods; i.e. a subgroup isomorphic to $\mathbb{Z}^{r}$.

Let $\Phi: M \rightarrow B(S)$ be a map which coincides with $q$ on the generic fiber.

(3) $\left(\lambda_{M}, \lambda_{B}\right)$ a polarization consisting of a

$$
\begin{array}{cc}
\lambda_{M}: M \rightarrow M^{\prime}:=\operatorname{Hom}_{S}\left(T, \mathbb{G}_{m, S}\right) & \text { group homomorphism } \\
\lambda_{B}: B \longrightarrow B^{\prime} & \text { polarization }
\end{array}
$$

such that the following conditions are satisfied:

(a) $\Phi^{\prime} \circ \lambda_{M}=\lambda_{B} \circ \Phi$

(b) $\left\langle m_{1}, \lambda_{M}\left(m_{2}\right)\right\rangle=\xi\left(\left\langle m_{2}, \lambda_{M}\left(m_{1}\right)\right\rangle\right)$ for all $m_{1} \in M_{1}, m_{2} \in M_{2}$.

(c) $\left\langle m, \lambda_{M}(m)\right\rangle \in \mathcal{I} P_{B \times B^{\prime}}$ for all $m \in M-\{0\}$,

where $\langle\cdot, \cdot\rangle: M \times M^{\prime} \rightarrow P_{B \times B^{\prime}}$ is the canonical pairing into the biextension given by the Poincaré bundle on $B \times{ }_{S} B^{\prime}$ lying over $\left(\Phi, \Phi^{\prime} \circ \lambda_{M}\right)$ and $\xi$ is the symmetry.

The meaning of the positivity condition (c) is the following: $\left\langle m, \lambda_{M}(m)\right\rangle$ gives rise to an $S$-section into the pull back of $P_{B \times B^{\prime}}$ to $S$ via $\left(\Phi, \Phi^{\prime} \circ\right.$ $\left.\lambda_{M}\right)$ and hence to a Cartier divisor of $S$. The positivity means that this divisor is effective on $S$ and congruent to $0 \bmod \mathcal{I}$. 
Theorem 3.5 (Chai, Faltings). In the situation of 3.4 there exists the quotient $G:=\widehat{G} / M$ which is a semi-abelian group scheme over $S$ with abelian generic fiber $G_{\eta}:=G \otimes_{S} K$ carrying a polarization $\lambda_{G}$ and semi-abelian $S_{0}$-group scheme $G \times{ }_{S} S_{0} \cong \widehat{G} \times{ }_{S} S_{0}$.

The proof uses the paper of Mumford as a blueprint. The main point is the construction of the relatively complete model. The situation here is much more complicated because of the abelian part $B$. Somehow one follows Mumford's method, but now in the relative situation $q: \widehat{G} \rightarrow B$. Locally over $B$, it looks like Mumford's situation and hence one can get a relatively complete model $\widehat{G} \subset$ $\widehat{P} \rightarrow B$ with a $B$-ample line bundle $E$. One has to twist the relative construction by an $S$-ample line bundle $N$ on $B$ which is provided by $\lambda_{B}$ from the given data in order to obtain a relatively complete model $\widehat{G} \subset \widehat{P} \rightarrow B$ and an $S$-ample line bundle $\widehat{L}=E \otimes q^{*} N$ on $\widehat{P}$ with $M$-linearization.

\section{RAYNAUD'S UNIFORMIZATION OF ABELIAN VARIETIES}

In this section, we go the opposite direction. Starting with a polarized abelian variety $G_{K}$ over a complete discretely valued field $K$ with ring of integers $R$, one wants to find the uniformization of $G_{K}$ resp. degeneration data in the sense of Mumford. The first steps for the uniformization were done by Néron [33] and Grothendieck [20].

Definition 4.1. Let $G_{K}$ be a smooth $K$-group variety. A Néron model $G$ of $G_{K}$ is a smooth separated $R$-scheme of finite type with generic fiber $G_{K}=G \otimes_{R} K$ satisfying the Néron mapping property:

For each smooth $R$-scheme $Z$ and each $K$-morphism $u_{K}: Z_{K} \rightarrow G_{K}$ there is a unique $R$-morphism $u: Z \rightarrow G$ extending $u_{K}$.

Theorem 4.2 (Néron). Any abelian variety $G_{K}$ admits a Néron model $G$.

For a proof in modern terms see [7]. $G$ is automatically a smooth $S$-group scheme. Let $G^{o}$ be the identity-component of the Néron model $G$. In general it does not commute with finite base field extension $K^{\prime} / K$; in particular it can be "small" for the given field in the sense that the canonical mapping $G^{o} \otimes_{R} R^{\prime} \rightarrow G^{\prime o}$ is not an isomorphism where $G^{\prime}$ denotes the Néron model of $G_{K} \otimes K^{\prime}$. If this is an isomorphism for any finite base field extension, the Néron model is called 
stable. A major step towards the uniformization is the Stable Reduction Theorem of Grothendieck [20].

Theorem 4.3 (Grothendieck). There exists a finite separable field extension $K^{\prime} / K$ such that the Néron model of $G \otimes_{K} K^{\prime}$ is semi-abelian.

After replacing $K$ by $K^{\prime}$ we may assume that the special fiber $G_{0}^{o}$ is an extension of an abelian variety by a torus $T_{0}$. Due to a theorem of Grothendieck [19], the torus lifts to a formal torus $\bar{T}$ in the formal completion $\bar{G}$ of $G^{o}$ with respect to the special fiber. So $\bar{G}$ is an extension of a formal abelian $R$-scheme $B$ by a formal torus $\bar{T}$

$$
\bar{T} \longrightarrow \bar{G} \longrightarrow B
$$

The generic fiber $\bar{G}_{\text {rig }} \subset G_{K}$ can be regarded as an open analytic subgroup of $G_{K}$. The formal torus is the torus of units $\bar{T}_{\text {rig }} \subset T_{K}$ inside an algebraic torus $T_{K}$ similar to $\{z ;|z|=1\} \subset \mathbb{G}_{m, K}$. It can be regarded as an open subdomain of the analytification of $T_{K}$. Let

$$
T_{K} \longrightarrow \widehat{G}:=T_{K} \times_{\bar{T}_{\text {rig }}} \bar{G}_{\text {rig }}
$$

be the push forward. The mapping $\bar{\varphi}: \bar{T}_{\text {rig }} \rightarrow G_{K}$ extends to a morphism $\varphi$.

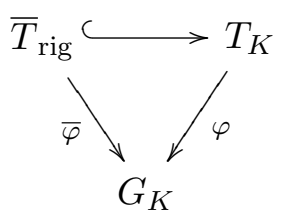

Therefore one obtains a mapping

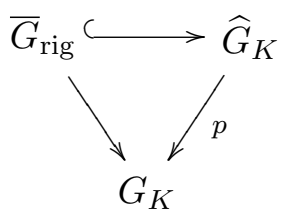

making $\widehat{G}_{K}$ to a quotient of $\widehat{G}_{K}$ by the kernel $M$ of $p$

$$
G_{K}=\widehat{G}_{K} / M \text {. }
$$

The extension of $\bar{\varphi}$ to $\varphi$ can be solved by looking at the decomposition of the Picard group of $G_{K}^{\prime} \times \mathbb{G}_{m, K}$ where $G_{K}^{\prime}$ is the dual of $G_{K}$ as it is explained in [5, $\S 1$ ] or by a direct calculation using the approximation techniques of $\S 6$; cf. [26, 
Prop. 3.1]. The surjectivity of $p$ follows from the fact that there is a bounded part $T_{K}(c)$ of $T_{K}$ satisfying $\bar{G}_{\text {rig }} \cdot T_{K}(c)=G_{K}$.

Theorem 4.4 (Raynaud). After a suitable finite separable field extension $K^{\prime} / K$, the abelian variety $G_{K}$ admits a representation

$$
G_{K}=\widehat{G}_{K} / M
$$

where $\widehat{G}_{K}$ is an extension of an abelian variety $B_{K}$ with good reduction by a split torus of rank $r$ and where $M$ is a lattice in $\widehat{G}_{K}$ of rank $r$.

The part that $B$ is an abelian variety with good reduction is explained in $\S 9$. In [4] this result was generalized to abelian varieties over algebraically closed fields which are complete with respect to valuations of rank 1 . The main difficulty was that there is no Néron model and no Stable Reduction Theorem of Grothendieck; both require a discrete valuation.

Chai and Faltings generalized Raynaud's uniformization for higher dimensional bases; the uniformization is the inverse functor to Mumford's construction; cf. [12, Theorem 6.2].

Theorem 4.5 (Chai, Faltings). Let $A$ be an excellent normal noetherian ring which is complete with respect to a reduced ideal $\mathcal{I} \subset A$ and let $K:=\operatorname{Frac}(A)$ its field of fractions. Set $S:=\operatorname{Spec}(A)$ and $S_{0}:=\operatorname{Spec}(A / \mathcal{I})$.

Let $G \rightarrow S$ be a semi-abelian group scheme over $S$ with abelian fiber $G_{\eta}$ and let $\lambda_{\eta}$ be a polarization on $G_{\eta}$. Assume that $G_{0}:=G \times_{S} S_{0}$ is an extension by a split torus of rank $r$. Then there exist

(1) a semi-abelian scheme $\widehat{G} \rightarrow S$ which is an extension of an abelian scheme $B \rightarrow S$ by a split torus $T \rightarrow S$ of rank $r$; this is equivalent to a group homomorphism $\Phi^{\prime}: M^{\prime}:=\operatorname{Hom}_{S}(T, B) \longrightarrow B^{\prime}(S)$ where $B^{\prime}$ is the dual of $B$,

(2) a set of periods $M \subset \widehat{G}_{\eta}$ of rank $r$ extending to a map $\Phi: M \rightarrow B(S)$

(3) a polarization $\left(\lambda_{M}, \lambda_{B}\right)$ in the sense of 3.4 ,

such that Mumford's construction to these data yields $\left(G, \lambda_{\eta}\right)$.

A discussion of the approach of Chai and Faltings and its relation to the methods of rigid geometry is given in [5, $\S 7]$. The main difference is how to find the lattice $M$. By the rigid method it is found via the uniformization as the kernel of the map $p: \widehat{G}_{\eta} \rightarrow G_{\eta}$. Chai and Faltings constructed it via the Fourier 
coefficients of the expansion of a nonzero section in a line bundle $L_{\eta}$ with respect to the characters when one restricts the line bundle to the formal subgroup $\bar{G}$ which is obtained from $G$ by completion with respect to the special fiber.

Theorem 3.5 and Theorem 4.5 are the main ingredients for constructing a toroidal compactification of the moduli space of principally polarized abelian varieties as was done by Chai and Faltings, cf. [12].

\section{Properness in FORMAL AND RIGID GeOMETRY}

The following three sections are devoted to explain the methods for analyzing the structure of abeloid varieties. The difficulties stem from the fact that there is no counterpart of the Stable Reduction Theorem 4.3.

Definition 5.1. A morphism of rigid spaces $f: X \rightarrow Y$ is called proper if it is separated and if there is an admissible covering $\left\{V_{i} ; i \in I\right\}$ of $Y$ by open affinoid subvarieties $V_{i}$ of $Y$ such that, for each $i \in I$, there exist two finite admissible coverings $\left\{U_{i, j}\right\}$ and $\left\{W_{i, j}\right\}$ of the inverse image $f^{-1}\left(W_{i}\right)$ by open affinoid subvarieties $U_{i, j}$ resp. $W_{i, j}$ such that $U_{i, j}$ is relatively compact in $W_{i, j}$ over $V_{i}$.

Given a morphism $W \rightarrow V$ of two affinoid varieties $V$ and $W$ and an open subvariety $U$ of $W$, one says $U$ is relatively compact in $W$ over $V$ (denoted $U \Subset_{V}$ $W$ ) if there exists a closed $V$-immersion of $W$ into an $n$-dimensional unit ball $\mathbb{D}_{V}^{n}(1)$ such that $U$ is sent into a ball with radius $c<1$ under the map $W \hookrightarrow$ $\mathbb{D}_{V}^{n}(1)$; i.e. one has a diagram

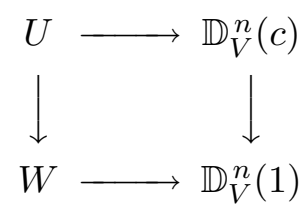

This definition is due to Kiehl; cf. [23, Def. 2.3]. It is made to be able to prove the Direct Image Theorem for coherent sheaves; cf. [23, Theorem 3.3]. For a long time, it was an open question if the composition of such proper morphisms is proper again. In the case of a discrete valued base field this problem was solved in [25] and later, for an arbitrary valued base field it was settled in [40].

Definition 5.2. A morphism $f_{K}: X_{K} \rightarrow Y_{K}$ of quasi-compact, quasi-separated rigid spaces is called proper in the formal sense if there exists a formal model 
$f: X \rightarrow Y$ of $f_{K}$ such that $f$ is proper; i.e. $f_{0}: X_{0} \rightarrow Y_{0}$ is proper.

Thereby we denote by $X_{n}$ the scheme $X \otimes_{R}\left(R / R \pi^{n+1}\right)$ for $n \in \mathbb{N}$.

It is easily shown that this definition is independent of the choice of the model. The main result of [25] is that the notion of properness in the rigid sense is equivalent to properness in the formal sense; thereby it became clear that the composition of proper rigid maps is proper as well. The main result in this context is the following theorem [25, Theorem 5.1] which we will discuss in this section in more detail.

Theorem 5.3. Consider the following situation

$$
\begin{array}{ll}
f: X \longrightarrow Y:=\operatorname{Spf}(B) \text { a morphism of admissble formal schemes, } \\
U \subset X & \text { an open formal subscheme, } \\
\overline{U_{0}} & \text { the schematic closure of } U_{0} \text { in } X_{0} .
\end{array}
$$

Assume that $\overline{U_{0}} \rightarrow Y_{0}$ is proper and that $U_{\text {rig }}$ is affinoid. Then there exist an admissible blowing up $X^{\prime} \longrightarrow X$ and an open formal subscheme $U^{\prime} \subset X^{\prime}$ such that

$$
\begin{array}{ll}
\overline{U_{0}^{\prime}} \longrightarrow \overline{U_{0}} & \text { is proper, } \\
U_{0}^{\prime} \supset \overline{\left(U \times_{X} X^{\prime}\right)_{0}} & \text { i.e. } U_{\text {rig }} \Subset_{Y_{\text {rig }}} U_{\text {rig }}^{\prime}, \\
U_{\text {rig }}^{\prime} & \text { is affinoid and } \\
\mathcal{O}\left(U_{\text {rig }}^{\prime}\right) \rightarrow \mathcal{O}\left(U_{\text {rig }}\right) \text { has dense image. }
\end{array}
$$

In particular $U_{\text {rig }}$ can be described as a subset of $U_{\text {rig }}^{\prime}$ via

$$
U_{\text {rig }}=\left\{x \in U_{\text {rig }}^{\prime} ;\left|f_{\rho}(x)\right| \leq c_{\rho} \text { for } \rho=1, \ldots, r\right\}
$$

where $f_{1}, \ldots, f_{r}$ are functions on $U^{\prime}$ and where $c_{\rho} \in \sqrt{\left|K^{\times}\right|}$for $\rho=1, \ldots, r$. Such domains are called Weierstraß domains.

As a corollary one obtains

Corollary 5.4. Let $f: X \rightarrow Y$ be a morphism of admissible formal schemes. The following are equivalent:

(a) $f$ is a proper formal morphism; i.e. $f_{0}: X_{0} \rightarrow Y_{0}$ is proper.

(b) $f_{\text {rig }}$ is a proper morphism of rigid analytic spaces. 
Let us briefly explain the idea of the proof for Theorem 5.3 in the discretely valued case. An important part is provided by the following algebraic facts; cf. [25, Def. 3.3 \& Prop. 5.4].

Definition 5.5. Let $Y$ be an affine noetherian scheme and let $X$ be a separated $Y$-scheme of finite type. Let $U$ be an open subscheme of $X$ and denote by $\bar{U}$ the schematic closure of $U$ in $X$. Let $\Delta$ be an effective Cartier divisor on $\bar{U}$.

(a) $\Delta$ is called positive on the boundary of $U$ if the support of $\Delta$ coincides with $\bar{U}-U$.

(b) $\Delta$ is called ample on the boundary of $U$ if $\Delta$ is positive on the boundary of $U$, the invertible sheaf $O_{\bar{U}}(\Delta)$ is generated by its global sections, and the restriction of $O_{\bar{U}}(\Delta)$ to the boundary $\bar{U}-U$ is (relatively) ample.

In the following a $Y$-compactification of a $Y$-scheme $U$ is a proper $Y$-scheme $V$ such that $U \subset V$ is open and schematically dense in $V$. A modification $X^{\prime}$ of an affine $Y$-scheme $X$ of finite type is a blowing up $X^{\prime} \rightarrow X$ of a subscheme $A$ of $X$ where $A$ is finite over $Y$.

Proposition 5.6. Let $X \rightarrow Y$ be a separated morphism of noetherian schemes where $Y$ is affine and let $U$ be an open subscheme of $X$ whose schematic closure $\bar{U}$ in $X$ is proper over $Y$. Then the following holds:

(a) $U$ admits a proper map to the affine $n$-space $\mathbb{A}_{Y}^{n}$ over $Y$ if and only if there exists a blowing-up $X^{\prime} \rightarrow X$ with center contained in $X-U$ which is a $Y$ compactification of $U$ such that there exists an effective Cartier divisor $\Delta$ on $X^{\prime}$ which is positive on the boundary of $U$ in $X^{\prime}$ such that $O_{X^{\prime}}(\Delta)$ is generated by its global sections.

(b) $U$ is a modification of an affine $Y$-scheme of finite type if and only if, in addition to (a), one can choose $\Delta$ to be ample on the boundary of $U$ in $X^{\prime}$.

(c) $U$ is affine if and only if, in addition to (a), one can choose $\Delta$ to be ample on $X^{\prime}$.

Generalizing results $[25, \S 4]$ of Goodman and Hartshorne [15], one can show the following assertion; cf. [25, Prop. 3.10].

Proposition 5.7. Consider the situation of Theorem 5.3. Assume that there exists an effective Cartier divisor on $\overline{U_{0}}$ which is ample on the boundary of $U_{0}$. Then the following holds:

(a) For any coherent formal $\mathcal{O}_{U}$-module $\mathcal{F}$ on $U$, the direct images $R^{n} f_{*} \mathcal{F}$ are 
coherent formal $\mathcal{O}_{Y}$-modules for $n \geq 1$.

(b) The ring of global sections $\mathcal{O}_{X}(U)$ is an admissible formal $\mathcal{O}_{Y}(Y)$-algebra.

(c) The Stein-factorization

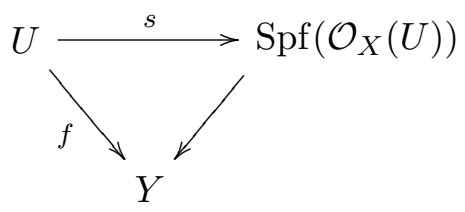

exists inside the category of admissible formal schemes; i.e. $\mathcal{O}_{X}(U)$ is an admissible $\mathcal{O}_{Y}(Y)$-algebra and $s$ is proper (in the formal sense).

The idea to prove Theorem 5.3 is the following.

Assume first that $U$ is affine. The consideration of this case is sufficient to prove Corollary 5.4. Then one can show that, after a suitable admissible blowing up, there exists a coherent open invertible ideal $\mathcal{I} \subset \mathcal{O}_{X}$ which induces an invertible ideal on $\overline{U_{0}}$ via $\mathcal{I} \cdot \mathcal{O}_{\overline{U_{0}}}$ with locus $\overline{U_{0}}-U_{0}$ and whose inverse is ample on the boundary of $U_{0}$. Namely using 5.6 , one can show that, after a suitable admissible blowing-up of $X$, there exists a Cartier divisor $\Delta$ on $\overline{U_{0}}$ which is ample on the boundary of $U_{0}$. Let $\mathcal{I}_{0} \subset \mathcal{O}_{\overline{U_{0}}}$ be the invertible ideal of $\Delta$ and let $\mathcal{I} \subset \mathcal{O}_{X}$ be the kernel $\operatorname{ker}\left(\mathcal{O}_{X} \rightarrow \mathcal{O}_{\overline{U_{0}}} / \mathcal{I}_{0}\right)$. Then replace $X$ by the formal blowing-up of $I$. Now look at

$$
\begin{array}{ll}
X^{(n)} \longrightarrow X & \text { the blowing up of the ideal } \mathcal{J}^{(n)}:=\left(\pi, \mathcal{I}^{n}\right) \\
W^{(n)} \subset X^{n} & \text { where } \mathcal{J}^{(n)} \text { is generated by } \mathcal{I}^{n} \\
U^{(n)} \subset W^{(n)} & \text { the union of connected components of } W^{(n)} \text { meeting } U
\end{array}
$$

Then the couple consisting of the invertible line bundle $\mathcal{L}:=\left(\mathcal{J}^{(n)}: \mathcal{I}^{n} \mathcal{O}_{X^{(n)}}\right)$ and the section $\iota: \mathcal{I}^{n} \mathcal{O}_{X^{(n)}} \hookrightarrow \mathcal{J}^{(n)}$ induced by the inclusion gives rise to an effective Cartier divisor which induces an invertible sheaf on the schematic closure $\overline{U_{0}^{(n)}}$ which is ample on the boundary of $U_{0}^{(n)}$. For sufficiently large $n$ this $U^{(n)}$ satisfies the claim. The latter mainly follows from Proposition 5.6 and 5.7.

The proof of the density is not given in [25]. Therefore we explain the proof of 
this statement in more details. Let $\lambda \in \mathbb{N}, \lambda \geq 1$ and set

$$
\begin{array}{ll}
A:=\operatorname{Spf}(R\langle\xi, \pi / \xi\rangle) & \\
T:=X \times A \supset W:=U \times A & \text { blowing-up of }(\mathcal{I}, \xi) \\
T^{\prime} \rightarrow T & \text { largest open subscheme where } \xi \in \mathcal{I O}_{T^{\prime}}, \\
T^{\prime} \supset W^{\prime} & \text { invertible line bundle on } T^{\prime} \\
\mathcal{L}:=\left((\mathcal{I}, \xi) \mathcal{O}_{T^{\prime}}: \mathcal{I O}_{T^{\prime}}\right) & \text { the inclusion }
\end{array}
$$

Then $(\mathcal{L}, \iota)$ gives rise to an effective Cartier divisor on schematic closure $\overline{W_{0}^{\prime}}$ which is ample on the boundary of $W_{0}^{\prime}$ in $\overline{W_{0}^{\prime}}$. Due to 5.7 the cohomology group $\mathrm{H}^{1}\left(W^{\prime}, \mathcal{O}_{T^{\prime}}\right)$ is finitely generated over $\mathcal{O}_{Y \times A}(Y \times A)$ and hence there exists an integer $\mu$ such that $\pi^{\mu}$ annihilates the $\pi$-torsion of $\mathrm{H}^{1}\left(W^{\prime}, \mathcal{O}_{T^{\prime}}\right)$.

Now consider some $f \in \Gamma\left(U, \mathcal{O}_{X}\right)$, then there exists an integer $n=n(f)$ such that, for any open subscheme $V$ of $X$ and for any $g \in \Gamma\left(U, \mathcal{I}^{n}\right)$, the section $g \cdot f \in \Gamma\left(U \cap V, \mathcal{O}_{X}\right)$ extends to a section of $\mathcal{O}_{X_{\mu+\lambda}}$ over $V$; cf. due to [17, EGA I, §1.3]. Now choose a trivialization

$$
\mathcal{I}^{n} \mid V^{i}=g_{i} \mathcal{O}_{V^{i}}
$$

for a finite open affine covering $\left(V^{1}, \ldots, V^{s}\right)$. Then we can write

$$
\xi^{n}=h_{i} \cdot g_{i} \text { over } W^{i}:=W^{\prime} \times_{X} V^{i} \text { for all } i=1, \ldots, s .
$$

Then there are sections $f_{i} \in \Gamma\left(W^{i}, \mathcal{O}_{T^{\prime}}\right)$ such that

$$
\xi^{n} \cdot f\left|W^{i}=f_{i}\right| W^{i} \bmod \pi^{\mu+\lambda+1} ;
$$

note that $W_{\xi}^{\prime}=U \times A_{\xi}$. The index $\xi$ indicates the open subscheme where $\xi$ is invertible. In particular the differences $\left(f_{i}-f_{j}\right)$ vanish over $\left(W_{\xi}^{i} \cap W_{\xi}^{j}\right)_{\mu+\lambda}$. Then there exists an integer $m \geq n$ such that

$$
\xi^{m} \cdot\left(f_{i}-f_{j}\right) \in \Gamma\left(W^{i} \cap W^{j}, \pi^{\mu+\lambda+1} \cdot \mathcal{O}_{T^{\prime}}\right) .
$$

Therefore the collection $\left(\xi^{m} \cdot f_{i}\right)_{i}$ gives rise to a section

$$
\left(\xi^{m} \cdot f_{i}\right)_{\mu+\lambda} \in \Gamma\left(W^{\prime}, \mathcal{O}_{T_{\mu+\lambda}^{\prime}}\right) .
$$

Next, look at the exact sequence

$$
\Gamma\left(W^{\prime}, \mathcal{O}_{T^{\prime}}\right) \longrightarrow \Gamma\left(W^{\prime}, \mathcal{O}_{T_{\mu+\lambda}^{\prime}}\right) \longrightarrow \mathrm{H}^{1}\left(W^{\prime}, \mathcal{O}_{T^{\prime}}\right) \stackrel{\pi^{\mu+\lambda+1}}{\longrightarrow} \mathrm{H}^{1}\left(W^{\prime}, \mathcal{O}_{T^{\prime}}\right)
$$


The image of $\left(\xi^{m} \cdot f_{i}\right)_{\mu+\lambda}$ in $\mathrm{H}^{1}\left(W^{\prime}, \mathcal{O}_{T^{\prime}}\right)$ is killed by $\pi^{\mu+\lambda+1}$, so it is annihilated by $\pi^{\mu}$, and, hence, the element $\pi^{\mu} \cdot\left(\xi^{m} \cdot f_{i}\right)_{\mu+\lambda}$ lifts to an element $F \in \Gamma\left(W^{\prime}, \mathcal{O}_{T^{\prime}}\right)$. Thus we obtain

$$
F-\pi^{\mu} \cdot \xi^{m+n} \cdot f \in \pi^{\mu+\lambda+1} \cdot \Gamma\left(W, \mathcal{O}_{T^{\prime}}\right) .
$$

Next we perform the base change induced by sending $\xi \longmapsto \rho$ where $\rho$ is an $(m+n)$-th root of $\pi$ and we see that $\pi^{\mu+1} \cdot f$ can be approximated by a section over $U^{\prime}(\rho):=W^{\prime} \times_{A} \operatorname{Spf}(R[\rho])$. Assume first $\rho \in R$. Then we have a canonical commutative diagram

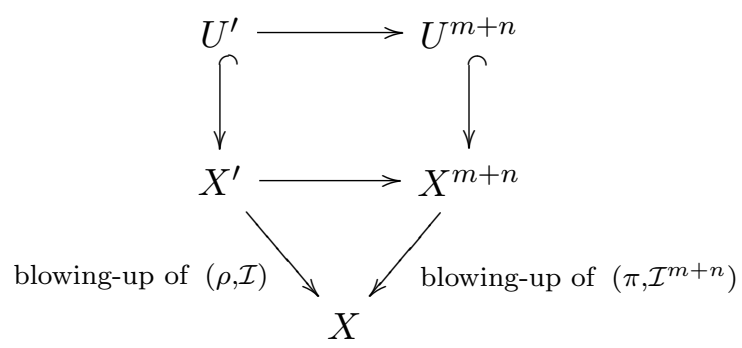

where $U^{\prime}=U(\rho)$ (resp. $\left.U^{m+n}\right)$ is the open subscheme of $X^{\prime}$ (resp. $X^{m+n}$ ) with $\rho \in \mathcal{I O}_{X^{\prime}}$ (resp. $\pi \in \mathcal{I}^{m+n} \mathcal{O}_{X^{m+n}}$ ). Thus we see that $f$ can be extended by $\pi^{-\mu-1} \cdot F$ which is a function over $U_{\text {rig }}^{\prime}$ up to a function in $\pi^{\lambda} \cdot \Gamma\left(U, \mathcal{O}_{X}\right)$. If $(m+n)$ is large enough, then $U^{\prime}$ is affinoid and $U \Subset_{Y} U^{\prime}$, since $U \Subset_{Y} U^{m+n}$. Since the ring of functions on $U_{\text {rig }}$ is finitely generated, say by $f_{1}, \ldots, f_{t} \in \mathcal{O}_{X}(U)$, we choose $U^{\prime}$ as above and functions $F_{1}, \ldots, F_{t}$ over $U^{\prime}$ which approximate $\pi^{\mu} f_{1}, \ldots, \pi^{\mu} f_{t}$ over $U$ up to $\pi^{\mu+1} \mathcal{O}_{X}$. So we can approximate any restricted power series in $f_{1}, \ldots, f_{t}$ by a polynomial in $F_{1} / \pi^{\mu}, \ldots, F_{t} / \pi^{\mu}$ up to an element in $\pi \Gamma\left(U, \mathcal{O}_{X}\right)$. Now we can iterate the process and thus we see that we can approximate any function on $U_{\text {rig }}$ up to $\pi^{\lambda}$ where we can choose $\lambda$ as large as we want.

Actually we have shown the assertion after a finite separable field extension; we assumed that a suitable root of $\pi$ lies in $R$. But one can easily show that the assertion " $\mathcal{O}_{X}\left(U_{\text {rig }}\right)$ is dense in $\mathcal{O}_{X}\left(U_{\text {rig }}^{\prime}\right)$ " can be checked after such a field extension. Then $U_{\text {rig }}$ is a Weierstraß domain in $U_{\text {rig }}^{\prime}$ due to $[3,7.3 .4 / 2]$.

Now let us briefly look at the general case which is more complicated.

Since the generic fiber of $U$ is affinoid, $U$ is obtained by a blowing up $U \rightarrow A$ of an open ideal on some affine admissible formal scheme $A$. So there exists an invertible sheaf $\mathcal{J} \subset \mathcal{O}_{U}$ of open ideals which is very ample with respect to $U \rightarrow A$. Assume that $\pi^{N} \in \mathcal{J}$ for some $N \in \mathbb{N}$. In the reduction modulo $\pi^{N+1}$ the 
invertible sheaf $\mathcal{J}$ induces a relatively very ample line bundle $\mathcal{L}_{N}$ and a morphism $\varphi: \mathcal{L}_{N} \rightarrow \mathcal{O}_{U_{N}}$ such that $\pi^{N}$ lies in the image of $\varphi$. Next we compactify the situation

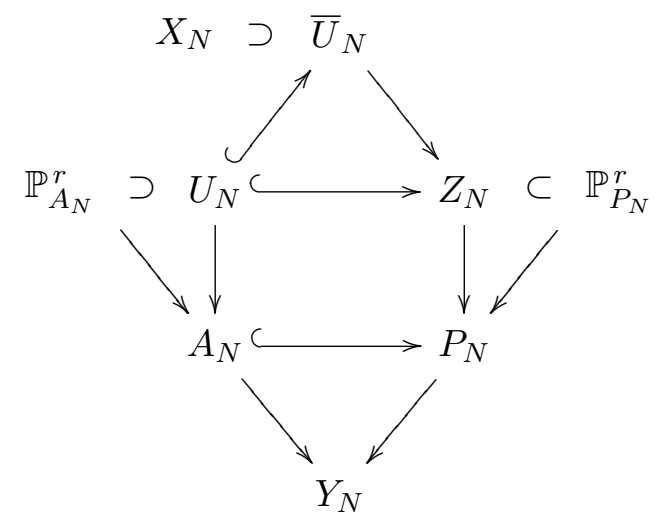

First we choose $A_{N} \hookrightarrow P_{N}$ as a projective $Y_{N}$-compactification with a very ample divisor $\Lambda_{N}$ with support $P_{N}-A_{N}$. Then we choose $Z_{N}$ as the schematic closure of $U_{N}$ in the product of the projective spaces $\mathbb{P}_{P_{N}}^{r}:=\mathbb{P}^{r} \times_{Y_{N}} P_{N}$ which contains $U_{N}$ via the embedding stemming from the line bundle $\mathcal{L}_{N}$. In particular the line bundle $\mathcal{L}_{N}$ extends to a line bundle $\overline{\mathcal{L}}_{N}$ which is relatively ample with respect to $Z_{N} \rightarrow P_{N}$. The morphism $\varphi$ extends to a morphism $\bar{\varphi}: \overline{\mathcal{L}}_{N} \rightarrow \mathcal{O}_{Z_{N}}$ after a suitable twisting $\overline{\mathcal{L}}_{N}$ with a power of $\Lambda_{N}$. Set $\mathcal{H}_{N} \subset \mathcal{O}_{\bar{U}_{N}}$ the image of $\bar{\varphi}$. Then there exist blowing-ups of $X$ and $Z_{N}$ such that $\bar{U}_{N}$ becomes a blowing-up $\bar{U}_{N} \rightarrow Z_{N}$ of $Z_{N}$ with center in $Z_{N}-U_{N}$ due to the flattening techniques of Raynaud. Thus we obtain an invertible sheaf $\mathcal{G}_{N}$ on $\bar{U}_{N}$ with locus in $\bar{U}_{N}-U_{N}$ which is very ample with respect to $\bar{U}_{N} \rightarrow Z_{N}$. Let $\Delta_{N}$ be the pull back of the Cartier divisor $\Lambda_{N}$ to $\bar{U}_{N}$ and let $\mathcal{I}_{N} \subset \mathcal{O}_{\bar{U}_{N}}$ be the ideal of its dual. Then the vanishing locus of $\mathcal{I}_{N}$ is $\bar{U}_{N}-U_{N}$. Now we lift everything to ideals of $\mathcal{O}_{X}$ via

$$
\begin{aligned}
& \mathcal{G}:=\operatorname{ker}\left(\mathcal{O}_{X} \rightarrow \mathcal{O}_{\bar{U}_{N}} / \mathcal{G}_{N}\right) \\
& \mathcal{H}:=\operatorname{ker}\left(\mathcal{O}_{X} \rightarrow \mathcal{O}_{\bar{U}_{N}} / \mathcal{H}_{N}\right) \\
& \mathcal{I}:=\operatorname{ker}\left(\mathcal{O}_{X} \rightarrow \mathcal{O}_{\bar{U}_{N}} / \mathcal{I}_{N}\right)
\end{aligned}
$$

Due to the construction there exist integers $r, s, t \in \mathbb{N}$ such that

$$
\mathcal{G}_{N}^{\otimes r} \otimes \mathcal{H}_{N}^{\otimes s} \otimes \mathcal{I}_{N}^{\otimes-t}
$$

are very ample invertible sheaves on $\bar{U}_{N}$. Then set

$$
\mathcal{S}:=\mathcal{G}^{r} \cdot \mathcal{H}^{s} \subset \mathcal{O}_{X}
$$


Now one considers

$$
\begin{array}{ll}
X^{(n)} \longrightarrow X & \text { the blowing up of the ideal } \mathcal{K}^{(n)}:=\left(\pi \mathcal{S}, \mathcal{I}^{n}\right) \\
W^{(n)} \subset X^{n} & \text { where } \mathcal{K}^{(n)} \text { is generated by } \mathcal{I}^{n} \\
U^{(n)} \subset W^{(n)} & \text { the union of connected components of } W^{(n)} \text { meeting } U
\end{array}
$$

Then $U^{n}$ satisfies the claim for sufficiently large $n \in \mathbb{N}$. This follows as in the special case. Namely, the invertible line bundle $\mathcal{T}:=\left(\mathcal{K}^{(n)}: \mathcal{I}^{n} \mathcal{O}_{X^{(n)}}\right)$ and the section $\iota: \mathcal{I}^{n} \mathcal{O}_{X^{(n)}} \hookrightarrow \mathcal{T}^{(n)}$ induced by the inclusion give rise to an effective Cartier divisor which induces an invertible sheaf on the schematic closure $\overline{U_{0}^{(n)}}$ being ample on the boundary of $U_{0}^{(n)}$.

\section{Artin's APproximation MEthod}

An important tool in rigid geometry is the approximation technique of Artin which was first noticed by Bosch in [2]. We will first state the result in terms of equations, later we will rephrase it in geometric terms.

Theorem 6.1 (Approximation Theorem). Let $K$ be a local field. Let $\zeta=$ $\left(\zeta_{1}, \ldots, \zeta_{n}\right)$ and $Y=\left(Y_{1}, \ldots, Y_{N}\right)$ be finitely many variables. For a constant $c \in \sqrt{\left|K^{\times}\right|}$of the divisible value group of $K$ let

$$
K\langle\zeta / c\rangle:=\left\{f=\sum a_{\nu} \zeta^{\nu} \in K[[\zeta]] ;\left|a_{\nu}\right| c^{\nu} \rightarrow 0\right\}
$$

be the algebra of power series converging on the polydisc $S:=\mathbb{D}^{n}(c)$ with radius c. Assume $c>1$ and let $\underline{f}:=\left(f_{1}, \ldots, f_{r}\right) \in K\langle\zeta / c, Y / c\rangle$ be a system of power series. Assume that there exists a solution $\widehat{y} \in K\langle\zeta\rangle$ of $\underline{f}(Y)=0$ converging on the polydisc $\widehat{S}:=\mathbb{D}^{n}(1)$ with radius 1 satisfying $|\widehat{y}| \leq 1$.

Then there exists $c^{\prime} \in \sqrt{\left|K^{\times}\right|}$with $1<c^{\prime}<c$ and a solution $y^{\prime} \in K\left\langle\zeta / c^{\prime}\right\rangle$ with $\underline{f}\left(y^{\prime}\right)=0$ arbitrarily close to $\widehat{y}$.

For a better understanding of the assertion we formulate it in geometric terms: Let $X=V(\underline{f}) \subset \mathbb{D}^{n+N}(c)$ be the closed subvariety associated to $\underline{f}$. A solution $\widehat{y}$ corresponds to an $S$-morphism $\widehat{\sigma}: \mathbb{D}^{n}(1) \longrightarrow X \cap \mathbb{D}^{n+N}(1)$. The assertion claims that for some $c^{\prime} \in\left|\sqrt{K^{\times}}\right|$with $1<c^{\prime}<c$ there exists an $S$-morphism $\sigma^{\prime}: \mathbb{D}^{n}\left(c^{\prime}\right) \rightarrow X$ arbitrarily close to $\widehat{\sigma}$.

If $X / S$ is smooth, the theorem is due to Elkik [11]. Namely Elkik states that 
there exists a constant $h>0$ depending only on the Jacobian $\left(\partial f_{i} / \partial Y_{j}\right)$ such that any nearby section $\widetilde{\sigma}: S \rightarrow \mathbb{D}^{n+N}(c)$ of a certain distance from $X$ depending on $h$ can be improved to a true section of $X$. For the proof of this fact one transforms the problem into the setting of integral equations over the valuation ring $R$ of $K$ and then one uses techniques similar to those in the classical Newton process.

For a proof of the approximation theorem in the smooth case, one first approximates the given section $\widehat{\sigma}$ by a nearby section $\widetilde{\sigma}: S^{\prime}:=\mathbb{D}^{n}\left(c^{\prime}\right) \rightarrow \mathbb{D}^{n+N}\left(c^{\prime}, c^{\prime \prime}\right)$ for some $1<c^{\prime}, c^{\prime \prime}<c$ and then one improves it to a true section $\sigma^{\prime}: S^{\prime} \rightarrow X$ by the method of Elkik.

Thus the approximation theorem is reduced to the following smoothening theorem by using the Lemma of Elkik [11, Lemme 1, p. 555].

Theorem 6.2 (Smoothening Theorem). Let $K, c, S, \widehat{S}$ and $\underline{f}=\left(f_{1}, \ldots, f_{r}\right) \in$ $K\langle\zeta / c, Y / c\rangle$ be as in 6.1. Set $X=V(\underline{f}) \subset \mathbb{D}^{n+N}(c)$. If $\overline{\widehat{\sigma}}: \widehat{S} \rightarrow X$ is an $S$-morphism with $\widehat{\sigma}(\widehat{S}) \subset X \cap \mathbb{D}^{n+N}(1)$, then there exists a commutative diagram

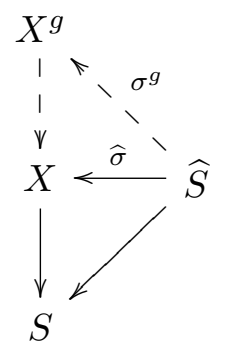

where $X^{g} \rightarrow S$ is smooth and $\sigma^{g}(\widehat{S})$ is relatively compact in $X^{g}$.

The proof of the smoothening theorem follows the lines of the classical theorem of Artin [1, Theorem 3.6/12] by using the Weierstraß Preparation Theorem [3, Theorem 5.2.2/1]. The approximation theorem was first considered by Bosch. In [2, Theorem 2], Bosch claimed a much stronger result than the one stated here, but his proof only works under the assumptions made here. The smoothening theorem was recently proved by Martin [28].

The approximation theorem becomes a very powerful tool in rigid geometry if one combines it with Theorem 5.3. For applications it is useful to generalize it and to formulate the final result in geometric terms. 
Theorem 6.3. Let $S$ be an affinoid space over a local field and let $\widehat{S} \subset S$ a Weierstraß domain such that $\widehat{S} \Subset S$ is relatively compact. Let $X$ be a quasicompact quasi-separated rigid $S$-space and $\lambda \in \mathbb{N}$. Assume that there exists an $S$-morphism $\widehat{\sigma}: \widehat{S} \rightarrow X$ such that $\widehat{\sigma}(\widehat{S}) \Subset_{S} X$. Then there exists an open subdomain $S^{\prime}$ of $S$ and an $S$-morphism $\sigma^{\prime}: S^{\prime} \rightarrow X$ with the following properties:

(1) $\widehat{S} \Subset S^{\prime} \Subset S$.

(2) $\left.\sigma^{\prime}\right|_{\widehat{S}} \equiv \widehat{\sigma} \bmod \pi^{\lambda}$ with respect to given integral models.

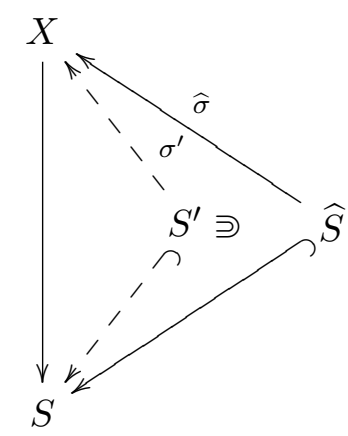

Proof. Due to Theorem 5.3, one can assume that $X$ is affinoid. One reduces the assertion to Theorem 6.1 by rewriting the assertion in terms of equations. Set

$$
\begin{aligned}
S & :=V\left(g_{1}, \ldots, g_{s}\right) \subset \mathbb{D}_{K}^{n}(c) \\
\widehat{S} & :=S \cap \mathbb{D}_{K}^{n}(1) \\
X & :=V\left(f_{1}, \ldots, f_{r}\right) \subset \mathbb{D}_{S}^{N}(1) \\
\widehat{\sigma}(\widehat{S}) & \subset \mathbb{D}_{S}^{N}(\varepsilon)
\end{aligned}
$$

with certain radii $c>1$ and $\varepsilon<1$. Let $\zeta_{1}, \ldots, \zeta_{n}$ resp. $Y_{1}, \ldots, Y_{N}$ be the coordinates of $\mathbb{D}_{K}^{n}$ resp. of $\mathbb{D}_{S}^{N}$. The section $\widehat{\sigma}$ induces elements

$$
\begin{aligned}
\widehat{y}_{\nu} & \in K\left\langle\zeta_{1}, \ldots, \zeta_{n}\right\rangle, \quad \nu=1, \ldots, N \\
\widehat{a}_{i j} & \in K\left\langle\zeta_{1}, \ldots, \zeta_{n}\right\rangle, \quad i=1, \ldots, r, j=1, \ldots, s
\end{aligned}
$$

satisfying

$$
\left\|\widehat{y}_{\nu}\right\|<1 \text { and } f_{i}(\widehat{y})=\sum_{j=1}^{s} \widehat{a}_{i j} g_{j} .
$$

Conversely, any system $\left(\left(\widehat{y}_{\nu}\right),\left(\widehat{a}_{i j}\right)\right)$ with these properties gives rise to a section $\widehat{\sigma}$. One may also assume $\left\|\widehat{a}_{i j}\right\|<1$ for all $i, j$ after multiplying the function 
$f_{1}, \ldots, f_{r}$ by a suitable constant. Now one considers the system of equations

$$
f_{i}=\sum_{j=1}^{s} Z_{i j} \cdot g_{j} \text { for } i=1, \ldots, r
$$

in the variables $Y$ and $Z$. This has a solution over $K\langle\zeta / c\rangle$. Then the assertion follows from Theorem 6.1.

It has far reaching consequences for the extension of isomorphisms and for the study of abeloid varieties as we will see in $\S 7$ and $\S 8$.

Corollary 6.4. Let $X^{1}$ and $X^{2}$ be rigid spaces as before. Let $U^{i} \subset X^{i}$ be open affinoid subdomains for $i=1,2$ such that each $U^{i} \Subset X^{i}$ is relatively compact. Assume that there is a morphism $\varphi: U^{1} \rightarrow U^{2}$. Then there exist open affinoid subdomains $W^{i} \subset X^{i}$ with $U^{i} \Subset W^{i}$ for $i=1,2$ and a morphism $\psi: W^{1} \rightarrow W^{2}$ which is arbitrarily close to $\varphi$. Furthermore, after a suitable shrinking of $W^{1}$ resp. $W^{2}$,

(a) if $\varphi$ is finite, so is $\psi$.

(b) if $\varphi$ is a closed immersion resp. an isomorphism, so is $\psi$.

Proof. The extension of an approximation $\psi: W^{1} \rightarrow W^{2}$ of $\varphi$ follows from Theorem 5.3 and 6.3. Since the induced map $\psi \mid U^{1}: U^{1} \rightarrow U^{2}$ is close to $\varphi$ the properties like finiteness, closed immersion resp. isomorphism are preserved. Then one can easily show that $\psi$ has the same properties as $\varphi$ after shrinking $W^{1}$ resp. $W^{2}$ in a suitable way.

\section{SEMI-STABle MOdELS FOR RIGID SPACES}

In the following assume that $K$ is a local field with a discrete valuation, let $R$ be its ring of integers and $\pi$ a uniformizing parameter. Starting with a smooth quasi-compact quasi-separated rigid space $X_{K}$, one cannot expect that $X_{K}$ has a smooth model over $\operatorname{Spf}(R)$ neither can one hope for an admissible open covering by domains isomorphic to polydiscs or polyannuli. For a covering by simple domains one has to allow a broader class of domains which is a combination of both classes mentioned before. The combination is meant here in terms of curve fibrations. This is possible in the étale topology of the analytic site. 
Definition 7.1. Let $X$ be a quasi-compact admissible formal $R$-scheme and let $X_{0}^{\sigma}$ for $\sigma=1, \ldots, s$ be the irreducible components of the special fiber $X_{0}$ of $X$. For $M \subset N:=\{1, \ldots, s\}, M \neq \emptyset$, we define

$$
X_{0}^{M}:=\bigcap_{\sigma \in M} X_{0}^{\sigma}
$$

as the scheme-theoretic intersection. $X$ is strictly semi-stable over $\operatorname{Spf}(R)$ if

(a) $X_{0}$ is geometrically reduced,

(b) $X_{0}^{\sigma}$ is a Cartier divisor on $X$ for all $\sigma \in N$ and

(c) $X_{0}^{M}$ is smooth over $k$ and equidimensional of dimension $\operatorname{dim} X-\# M$ for all $M \subset N, M \neq \emptyset$.

If $X$ is strictly semi-stable over $\operatorname{Spf}(R)$, then $X$ is a regular scheme due to condition (b) and the generic fiber $X_{\text {rig }}$ is smooth over $\operatorname{Sp}(K)$. One can show:

Remark 7.2. Let $X$ be a quasi-compact, admissible formal $R$-scheme. The following are equivalent:

(a) $X$ is strictly semi-stable.

(b) Every closed point $x \in X_{0}$ of the special fiber has an open neighborhood which for some $r \in \mathbb{N}$ is formally smooth over the formal scheme

$$
\operatorname{Spf}\left(R\left\langle\xi_{1}, \ldots, \xi_{r}\right\rangle /\left(\xi_{1} \cdot \ldots \cdot \xi_{r}-\pi\right)\right) .
$$

Definition 7.3. Let $f: S^{\prime} \rightarrow S$ be a morphism of quasi-compact admissible formal $R$-schemes. The map $f$ is called rig-étale cover if $f$ is a composition $S^{\prime} \rightarrow S^{\dagger} \rightarrow S$ of an admissible formal blowing-up $S^{\dagger} \rightarrow S$ by a morphism $S^{\prime} \rightarrow S^{\dagger}$ which is quasi-finite, flat and surjective, as well as rig-étale, i.e. étale on the associated rigid spaces $S_{\text {rig }}^{\prime} \rightarrow S_{\text {rig }}^{\dagger}$.

This implies that $f_{\text {rig }}: S_{\text {rig }}^{\prime} \rightarrow S_{\text {rig }}$ is étale, surjective. Conversely every étale surjective morphism $f_{K}: S_{K}^{\prime} \rightarrow S_{K}$ of quasi-compact, quasi-separated rigid spaces has a model $f$ which is rig-étale.

The main result in this context is:

Theorem 7.4. Let $X$ be a quasi-compact admissible formal scheme such that the associated rigid space $X_{\text {rig }}$ is smooth over $\operatorname{Sp}(K)$. Then there exists a finite separable field extension $K^{\prime}$ of $K$ with ring of integers $R^{\prime}$ and a rig-étale cover $X^{\prime} \rightarrow X \otimes_{R} R^{\prime}$ of quasi-compact formal $R^{\prime}$-schemes such that $X^{\prime}$ is strictly semistable over $\operatorname{Spf}\left(R^{\prime}\right)$. 
In terms of rigid spaces one can rewrite it in the following terms.

Corollary 7.5. Let $X_{K}$ be a smooth rigid space over $\operatorname{Sp}(K)$ assumed to be quasicompact and quasi-separated. Then there exists a finite separable field extension $K^{\prime}$ of $K$ with ring of integers $R^{\prime}$ and an étale surjective morphism $X_{K}^{\prime} \rightarrow X_{K}$ of a quasi-compact quasi-separated rigid $K^{\prime}$-space $X_{K}^{\prime}$ which admits a strictly semi-stable formal $R^{\prime}$-model $X^{\prime}$.

A proof of this result was sketched in [26]; details were worked out by Hartl [21, Theorem 1.4]. We give a brief sketch of the proof. Let $X$ be a formal model of the given rigid space $X_{K}$. Locally on $X$, one fibers $X$ over a formal scheme $Y$ such that all fibers are one-dimensional and then one uses induction on the dimension $d$ of $X_{K}$. Since $X_{K}$ is rig-smooth, it can locally be fibered over an admissible open set $Y_{K}$ of the $(d-1)$-dimensional unit ball $Y \subset \mathbb{D}_{K}^{d-1}$ such that $X_{K} \rightarrow Y_{K}$ is a smooth curve fibration. The map $X_{K} \rightarrow Y_{K}$ has a formal model $X \rightarrow Y$ such that the map is flat and, hence, a rig-smooth curve fibration. We are free to perform a rig-étale base change to $Y$. So, using the Reduced Fiber Theorem 2.6, we may assume that $X \rightarrow Y$ is flat with geometrically reduced fibers. By formal patching and using the extension of morphisms 6.4, one can compactify this fibration to a projective, rig-smooth curve fibration $P \rightarrow Y$ with geometrically reduced fibers such that $X \subset P$ is an open subscheme; cf. [26, Theorem 5.3]. After allowing a rig-étale base change, one can assume that $P \rightarrow Y$ is punctured by sections making $X_{\text {rig }} \rightarrow Y_{\text {rig }}$ into a punctured stable curve over $Y_{\text {rig. }}$. Using the properness of the moduli space of stable punctured curves, we can replace $Y$ by a rig-étale cover such that $P_{\text {rig }}$ extends to a stable punctured curve $C \rightarrow Y$; i.e. $C_{\text {rig }} \stackrel{\sim}{\longrightarrow} P_{\text {rig. }}$. As in [10] one shows that the morphism $C_{\text {rig }} \stackrel{\sim}{\longrightarrow} P_{\text {rig }}$ extends to a morphism $C \rightarrow P$ after an admissible blowing-up of $Y$. Thus we can replace $P$ by $C$; cf. [26, Theorem 5.9] and hence we have obtained a relative version of the desingularization.

Theorem 7.6. Let $f_{K}: X_{K} \rightarrow Y_{K}$ be a smooth rigid curve fibration. Then, étale locally on $Y_{K}$ and locally on $X_{K}$, there exists a smooth projective $Y_{K^{-}}$ compactification.

Moreover, étale locally on $X_{K}$, there exists an ordinary model of $f_{K}$; i.e. a formal model $f: X \rightarrow Y$ of $f_{K}$ such that $f$ is flat with geometrically reduced fibers and its singularities are at most ordinary multiple points. 
Now the induction hypothesis allows us to replace $Y$ by a strictly semi-stable formal $R$-scheme. This means that the special fiber $Y_{0}$ is a divisor with strict normal crossings. Since $C_{\text {rig }}$ is smooth over $Y_{\text {rig }}$ we see that the singularities of $C$ lie in the special fiber and are given by equations of the form

$$
\zeta \cdot \eta=t_{1}^{n_{1}} \cdot \ldots \cdot t_{r}^{n_{r}} .
$$

These can be resolved explicitly. Since $X$ is an open subscheme of $C$, the claim 7.4 follows.

\section{Abeloid Varieties}

A compact connected complex Lie group of dimension $g$ can be represented as a quotient $\mathbb{C}^{g} / \Lambda$ of the affine vector group $\mathbb{C}^{g}$ by a lattice $\Lambda$ of rank $2 g$. From the multiplicative point of view, it can be described as a quotient $\left(\mathbb{C}^{*}\right)^{g} / M$ of the affine torus $\left(\mathbb{C}^{*}\right)^{g}$ by a multiplicative lattice $M$ of rank $g$. In the rigid case, the situation is more complicated because of the phenomena of good and split reduction which in general occur in a twisted form.

Definition 8.1. An abeloid variety is a group object in the category of rigid analytic spaces whose underlying variety is smooth, proper and connected.

The fundamental example of such a rigid group $G_{K}$ is the analytic quotient $G_{K}=\widehat{G}_{K} / M_{K}$ where $\widehat{G}_{K}$ is an extension of an abeloid group $B_{K}$ with good reduction by an affine torus $T_{K}$, and where $M_{K}$ is a lattice in $\widehat{G}_{K}$ of $\operatorname{rank} \operatorname{dim}\left(T_{K}\right)$; cf. Example 3.1. The main result of [26] is that any abeloid variety is of the form $\widehat{G}_{K} / M_{K}$ after a suitable extension of the base field as described in the example. This is a generalization of Grothendieck's Stable Reduction Theorem [20, I, Exp. IX, 3.5] as well as of the rigid uniformization of abelian varieties to the analytic case; cf. Theorem 4.4. More generally, one can study bounded rigid groups. A connected smooth rigid group is called bounded if it is quasi-compact and commutative; for example a proper smooth rigid group is bounded.

In the following assume that $K$ is a local field with a discrete valuation. Let $R$ be its ring of integers and $\pi$ a uniformizing parameter. We would like to mention that the following results are also true for local fields with an arbitrary valuation of rank 1, but they are not written. In [26] they were proved only for fields with a discrete valuation since at that time the extension property for affinoid 
subdomains 5.3 and consequently for morphisms 6.4 were only established in the case of discrete valuations. Due to the result of Temkin [40] these facts are true in general. Therefore the arguments of [26] work in general as well.

Theorem 8.2. Let $G_{K}$ be a smooth rigid group assumed to be bounded and connected. After a suitable finite separable field extension of $K$, there exists an open connected analytic subgroup $\bar{G}_{K}$ of $G_{K}$ which admits a smooth formal model $\bar{G}$ over $R$. Moreover, there exists a rigid analytic group homomorphism $\Phi: T_{K} \rightarrow G_{K}$ of a split affine torus $T_{K}=\mathbb{G}_{m, K}^{r}$ to $G_{K}$ such that:

(i) the restriction $\bar{\Phi}$ of $\Phi$ to the open analytic subtorus $\bar{T}_{K}=\overline{\mathbb{G}}_{m, K}^{r}$ of units of $T_{K}$ induces a closed immersion $\bar{T}=\overline{\mathbb{G}}_{m, R}^{r} \hookrightarrow \bar{G}$ which splits in the maximal formal torus of $\bar{G}$,

(ii) there is a bounded part $T_{K}(b)$ of $T_{K}$ such that $\Phi\left(T_{K}(b)\right) \cdot \bar{G}_{K}=G_{K}$.

The subgroup $\bar{G}_{K}$ of $G_{K}$ is uniquely determined by these conditions; in particular it is the largest open connected analytic subgroup of $G_{K}$ which admits a smooth model over $R$.

The open subtorus of units $\overline{\mathbb{G}}_{m, K}$ of $\mathbb{G}_{m, K}$ is the open analytic subset of $\mathbb{G}_{m, K}$ consisting of the points $z$ with $|z|=1$. Denote by $\widehat{G}_{K}$ the push-forward of $\bar{G}_{K}$ via the morphism $\bar{T}_{K} \hookrightarrow T_{K}$. So we get a surjective homomorphism $p: \widehat{G}_{K} \rightarrow G_{K}$ of rigid groups via $\Phi$. The kernel $M_{K}=\operatorname{ker}(p)$ is a discrete subgroup of $\widehat{G}_{K}$.

Corollary 8.3. In the situation of Theorem 8.2, the group $G_{K}$ admits a representation $G_{K} \simeq \widehat{G}_{K} / M_{K}$, where $\widehat{G}_{K}$ is an extension of the generic fiber of the smooth formal group scheme $B=\bar{G} / \bar{T}$ by an affine torus $T_{K}$, and where $M_{K}$ is a lattice of $\widehat{G}_{K}$ of rank equal to the rank of $\operatorname{Hom}\left(\mathbb{G}_{m, K}, G_{K}\right)$. The latter can be less than the rank of $\operatorname{Hom}\left(\overline{\mathbb{G}}_{m, K}, G_{K}\right)$

A formal Néron model of $G_{K}$ is the largest open analytic subgroup $N_{K}$ which admits a smooth quasi-compact formal model over $R$; it depends on the base field $K$. One can show as in the algebraic case [7] that any bounded group has a formal Néron model; cf. [8]. A formal Néron model is called stable if its 1-component is compatible with finite separable field extensions.

Corollary 8.4. For any bounded group $G_{K}$ there exists a finite separable field extension $K^{\prime} / K$ such that the formal Néron model of $G_{K} \otimes_{K} K^{\prime}$ is stable. 
The proof requires several auxiliary facts which will be sketched in the following. The first ingredient is a statement about group generation [26, Theorem $1.2]$.

8.5 (Group Generation).

Let $G_{K}$ be a smooth commutative rigid group. Let $X$ be a quasi-compact connected smooth formal $R$-scheme and let $o \in X_{\text {rig }}$ be a rational point. Let $u_{K}: X_{\text {rig }} \rightarrow G_{K}$ be a rigid morphism sending $o$ to the unit element. Then $X$ generates a smallest locally closed subgroup $H_{K}$ of $G_{K}$ via $u_{K}$ which admits a smooth formal $R$-model $H$ such that $u_{K}$ factors through $H_{K}$.

In particular, $u_{K}$ extends to a morphism $u: X \rightarrow H$. Moreover, if $d$ is the dimension of $G_{K}$, the multiplication map $u^{2 d}: X^{2 d} \rightarrow H$ is surjective.

If $X_{K}^{\prime}$ is a rigid space with $X_{\text {rig }} \Subset X_{K}^{\prime}$ and if $u_{K}$ extends to a rigid map $u_{K}^{\prime}: X_{K}^{\prime} \rightarrow G_{K}$, then $H_{\text {rig }}$ is relatively compact in $G_{K}$. Furthermore, if $X_{0}$ is a rational variety, $H$ has linear reduction.

If $X$ is not connected, we allow to perform a finite field extension to puncture the components $\left(X^{i} ; i=1, \ldots, n\right)$ by rational points $x_{i} \in X^{i}$ and define $\langle X, u\rangle$ as the group generated by $\left(X^{i}, u \cdot u\left(x_{i}\right)^{-1}\right)$ for $i=1, \ldots, n$. It is easy to see that the definition does not depend on the choices of the points.

As a consequence, by using Theorem 5.3, one obtains the following:

Corollary 8.6. A stable formal Néron model of an abeloid variety has semiabelian reduction.

As a second important fact, one has to exhibit the structure of maps from curves to groups. This starts with the following result; cf. [26, Prop. 3.1].

8.7 (Extension of multiplicative group homomorphisms).

Let $\bar{\varphi}: \overline{\mathbb{G}}_{m, K} \rightarrow G_{K}$ be a homomorphism of rigid groups. Assume that the image of $\bar{\varphi}$ is relatively compact in $G_{K}$. Then $\bar{\varphi}$ extends to a homomorphism $\varphi: \mathbb{G}_{m, K} \rightarrow G_{K}$.

This can be generalized to the following [26, Theorem 4.1]:

8.8 (Maps from Curves to Groups).

Let $C$ be a connected smooth affine formal curve which is semi-stable and which has precisely one singular point $x_{0}$, and let $u: C_{K} \rightarrow G_{K}$ be a rigid map. Denote 
by $\bar{C}$ the smooth part of $C$ over $\operatorname{Spf}(R)$. Let $\bar{G}_{K}:=\left\langle\bar{C}_{\text {rig }}, u\right\rangle \subset G_{K}$ be the subgroup generated by the smooth part, and let $\bar{G}$ be the smooth formal model of $\bar{G}_{K}$. Furthermore, let $\xi: C_{K} \rightarrow \mathbb{G}_{m, K}$ be a rigid morphism which restricts to a coordinate on the formal fiber $C_{K}\left(x_{0}\right)$ of the double point $x_{0}$.

Then there exists a unique rigid group homomorphism $\varphi: \mathbb{G}_{m, K} \rightarrow G_{K}$ with $\varphi\left(\overline{\mathbb{G}}_{m, K}\right) \subset \bar{G}_{K}$ such that $u$ factors

$$
u(x)=(\varphi \circ \xi)(x) \cdot \bar{u}(x) \cdot g \text { for } x \in C_{K},
$$

where $\bar{u}: C_{K} \rightarrow \bar{G}_{K}$ is a rigid morphism and where $g \in G_{K}$ is a point. If $\varphi$ is not trivial, one can write $\varphi=\psi^{n}$ for a rigid homomorphism $\psi: \mathbb{G}_{m, K} \rightarrow G_{K}$ where $n$ is the order of $\varphi$.

Adding up the tori, one can find a morphism of rigid groups

$$
\Phi: \mathbb{G}_{m, K}^{r} \longrightarrow G_{K}
$$

which restricts to a closed immersion

$$
\bar{\Phi}: \overline{\mathbb{G}}_{m, K}^{r} \longrightarrow \bar{G}
$$

such that any morphism of rigid groups $\varphi: \mathbb{G}_{m, K} \rightarrow G_{K}$ factors through $\Phi$.

The most important tool is provided by [26, Theorem 5.9]; see also Theorem 7.4.

8.9 (Stable reduction of relative curves).

Let $f_{K}: X_{K} \rightarrow S_{K}$ be a smooth rigid curve fibration. Then, étale locally on $X_{K}$ and $S_{K}$, there exists an ordinary model of $f_{K}$; i.e., a formal model $f: X \rightarrow S$ of $f_{K}$ such that $f$ is flat with geometrically reduced fibers such that its singularities are at most ordinary multiple points.

By using these facts, we will sketch the proof of the structure theorem.

There exists an étale cover $u_{K}: X_{K} \rightarrow G_{K}$ of $G_{K}$ by a smooth quasi-compact rigid space admitting a curve fibration $X_{K} \rightarrow S_{K}$ which has a model $X \rightarrow S$ of admissible formal schemes which is flat with geometrically reduced fibers such that their singularities are at most ordinary multiple points. Furthermore, one can assume that $S$ has a geometrically reduced special fiber over $\operatorname{Spf}(R)$. Let $X^{*}$ be the smooth part of $X / S$. After base change by a suitable rig-étale cover, one can assume that $X^{*} \rightarrow S$ admits a section and that its fibers are connected. 
Notice that $X^{*}$ is open dense in each fiber of $X / S$. Let $\bar{X}$ be the smooth part of $X / R$. Then set

$$
\bar{G}_{K}:=\left\langle\bar{X}_{\text {rig }}, u_{K}\right\rangle \subset G_{K} .
$$

So $\bar{G}_{k}$ has a smooth model $\bar{G}$ over $R$ due to 8.5. One may assume that all the tori which appear by looking at the restriction of $u_{K}$ to the fibers of $X_{K} \rightarrow S_{K}$ are already contained in $\bar{G}_{K}$. Now one shows by an extension property of rational morphisms that

$$
u\left(x_{1}\right) \cdot u\left(x_{2}\right)^{-1} \in \bar{G}_{\text {rig }} \text { for all }\left(x_{1}, x_{2}\right) \in X^{*} \times_{S} X^{*} .
$$

By the property of mappings from curves to groups 8.8, one can show

$$
u\left(x_{1}\right) \cdot u\left(x_{2}\right)^{-1} \in \Phi\left(\mathbb{G}_{m, K}^{r}\right) \cdot \bar{G}_{\text {rig }} \text { for all }\left(x_{1}, x_{2}\right) \in X \times_{S} X .
$$

Moreover one can estimate a bounded part $\Phi\left(\mathbb{G}_{m, K}^{r}(b)\right) \Subset \Phi\left(\mathbb{G}_{m, K}^{r}\right)$ which is necessary for the last assertion. As a final step, one shows

$$
u\left(x_{1}\right) \cdot u\left(x_{2}\right)^{-1} \in \Phi\left(\mathbb{G}_{m, K}^{r}\right) \cdot \bar{G}_{\text {rig }} \text { for all } x_{1}, x_{2} \in X
$$

by looking at a sequence of points $x_{1}, y_{1}, \ldots, y_{n}, x_{2}$ such that consecutive points belong to fibers of the curve fibration $X / S$ or of the base $S$.

The group $\bar{G}_{K}$ has the following universal properties:

(a) Let $Z$ be a connected smooth admissible formal $R$-scheme and consider a rigid morphism $u_{K}: Z_{K} \rightarrow G_{K}$. If there is a rig-point $z$ of $Z$ with $u_{K}(z) \in \bar{G}_{K}$, then $u_{K}$ factors through $\bar{G}_{K}$.

In particular, $\bar{G}_{K}$ is the 1-component of the formal Néron model of $G_{K}$.

(b) Let $C$ be a formal semi-stable affine curve with precisely one singular point $x_{0}$ assumed to be a double point. Let $\xi: C_{\text {rig }} \rightarrow \mathbb{G}_{m, K}$ be a rigid map which restricts to a coordinate on the formal fiber $C_{K}\left(x_{0}\right)$ of $x_{0}$.

Let $u: C_{\text {rig }} \rightarrow G_{K}$ be a rigid morphism. Then there exists a rigid group homomorphism $\varphi: \mathbb{G}_{m, K} \rightarrow G_{K}$ such that $u$ factors

$$
u=(\varphi \circ \xi) \cdot \bar{u} \cdot g
$$

where $\bar{u}: C_{\text {rig }} \rightarrow \bar{G}_{K}$ is a rigid morphism and where $g \in G_{K}$.

The properties mainly follow from our results about mappings from curves to groups 8.8.

In the case of abeloid varieties one obtains the final structure theorem. 
Theorem 8.10. Let $G_{K}$ be an abeloid variety. After a suitable finite separable field extension, $G_{K}$ admits a Raynaud representation as in Example 3.1:

(a) There exists a largest open connected analytic subgroup $\bar{G}_{K}$ of $G_{K}$ which admits a smooth formal model $\bar{G}$ being an extension of a formal abelian scheme $B$ over $\operatorname{Spf}(R)$ by a formal torus $\bar{T}$.

(b) The canonical maps (restriction resp. reduction)

$$
\operatorname{Hom}\left(\mathbb{G}_{m, K}, G_{K}\right) \stackrel{\sim}{\longrightarrow} \operatorname{Hom}\left(\overline{\mathbb{G}}_{m, K}, \bar{G}_{K}\right) \stackrel{\sim}{\longrightarrow} \operatorname{Hom}\left(\mathbb{G}_{m, k}, \bar{G}_{k}\right)
$$

are bijective. In particular there exists an analytic group homomorphism from a split affine torus $\Phi: T_{K} \rightarrow G_{K}$ such that

(i) $\left.\Phi\right|_{\bar{T}_{K}}$ is an isomorphism between $\bar{T}$ and the maximal torus of $\bar{G}$,

(ii) there is a bounded part $T_{K}(\alpha)$ of $T_{K}$ with $\Phi\left(T_{K}(\alpha)\right) \cdot \bar{G}_{K}=G_{K}$.

(c) Let $\widehat{G}_{K}$ be the push-forward of $\bar{G}_{K}$ via the morphism $\bar{T}_{K} \hookrightarrow T_{K}$. The map $\Phi$ induces a group morphism $p: \widehat{G}_{K} \rightarrow G_{K}$. The kernel of $p$ is a lattice $M_{K}$ of rank $\operatorname{dim}\left(\bar{T}_{K}\right)$ and $G_{K}$ is the quotient of $\widehat{G}_{K}$ by $M_{K}$.

Such $a G_{K}$ can be described by the following diagram:

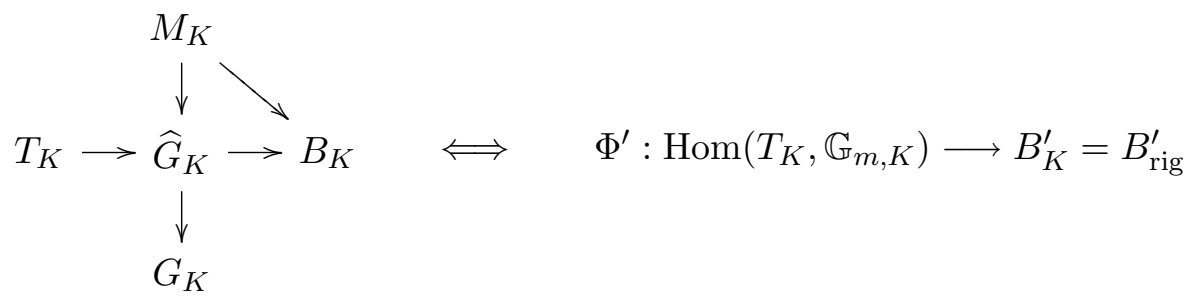

\section{PicARd VARIETIES}

The representability of the Picard functor for smooth rigid spaces was first settled by Bosch and the author for abeloid varieties which are uniformized; cf. [5]. Later in [22] Hartl and the author treated the general case. The case of abeloid varieties is probably more interesting since it is related to the question for necessary and sufficient conditions for an abeloid variety to be algebraic. Let 
us recall this case first. Start with the following situation

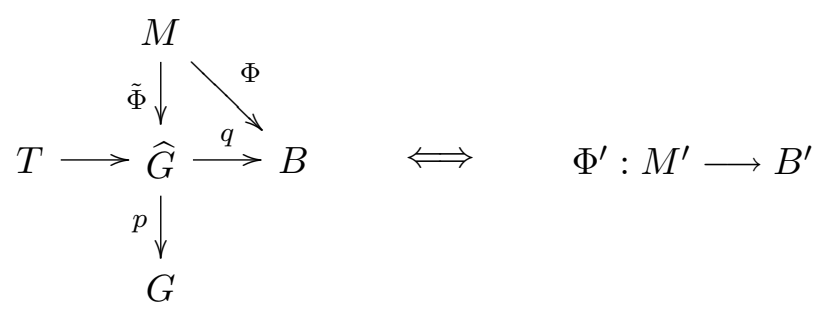

where we omit the index $K$ when indicating rigid analytic objects and where

$T$ is a split affine torus of rank $r$

$B \quad$ is the generic fiber of a formal abelian scheme over $\operatorname{Spf}(R)$

$B^{\prime} \quad$ is the generic fiber of the dual of $B$

$\widehat{G} \quad$ is an extension of $B$ by $T$

$M \quad$ is a constant group scheme isomorphic to $\mathbb{Z}^{r}$

$M^{\prime}$ is the character group $\operatorname{Hom}\left(T_{K}, \mathbb{G}_{m, K}\right)$ of $T$

$\tilde{\Phi}$ defines $M$ as a lattice in $\widehat{G}$ lifting the group morphism $\Phi$

$\Phi^{\prime}$ describes the extension $\widehat{G}$ in terms of line bundles

$G \quad$ is the quotient of $\widehat{G}$ with respect to $M$

Such data are equivalent to a commutative diagram

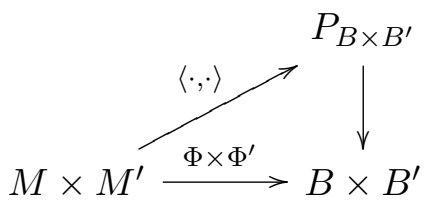

where

$$
\begin{array}{ll}
P_{B \times B^{\prime}} & \text { is the Poincaré bundle } \\
\Phi: M \rightarrow B & \text { is a group homomorphism } \\
\Phi^{\prime}: M^{\prime} \rightarrow B^{\prime} & \text { is a group homomorphism } \\
B^{\prime} & \text { is the dual of } B \\
\langle\cdot, \cdot\rangle: M \times M^{\prime} \rightarrow P_{B \times B^{\prime}} & \text { is the non-degenerate bilinear form }
\end{array}
$$

Due to the self duality $B^{\prime \prime}=B$, this description is symmetric in $\left(B, \Phi^{\prime}, \Phi\right)$ and 
$\left(B^{\prime}, \Phi, \Phi^{\prime}\right)$. Thus it gives rise to the dual diagram

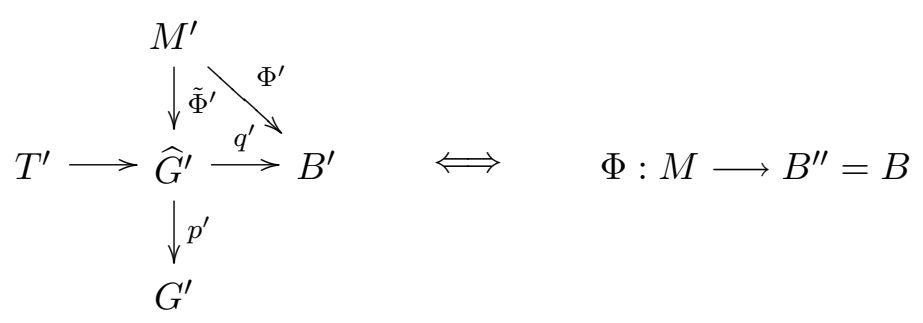

and, hence, it defines an abeloid variety $G^{\prime}$.

One can describe line bundles $L$ on $G$ in terms of $M$-linearizations. An $M$ linearization can be described as a triple $(N, \lambda, r)$ where

$N$ is a line bundle on $B$ with $p^{*} L=q^{*} N$

$\lambda: M \rightarrow M^{\prime} \quad$ is a group homomorphism

$r: M \rightarrow \Phi^{*} N$ is a trivialization

satisfying for all $m_{1} \in M_{1}, m_{2} \in M_{2}$

$$
\Phi^{\prime} \circ \lambda=\varphi_{N} \circ \Phi \text { and } r\left(m_{1}+m_{2}\right) \otimes r\left(m_{1}\right)^{-1} \otimes r\left(m_{2}\right)^{-1}=\left\langle m_{1}, \lambda\left(m_{2}\right)\right\rangle
$$

where $\varphi_{N}: B \rightarrow B^{\prime}, b \mapsto \tau_{b}^{*} N \otimes N^{-1}$ is the canonical map. The identity for the section $r$ has to be read in

$$
\mu^{*} N \otimes p_{1}^{*} N^{-1} \otimes p_{2}^{*} N^{-1} \cong\left(\operatorname{id}_{B} \times \varphi_{N}\right)^{*} P_{B \times B^{\prime}}
$$

where $p_{i}: B \times B \rightarrow B$ is the $i$-th projection and $\mu: B \rightarrow B \times B$ is the group law. The line bundle $L$ is translation invariant if and only if $\lambda=0$ and $N$ is translation invariant; i.e. $\varphi_{N}$ is constant and equals the unit element of $B^{\prime}$. Therefore a translation invariant line bundle $L$ is equivalent to a point in $\widehat{G}^{\prime}$ up to a translation by a point of $\tilde{\Phi}^{\prime}\left(M^{\prime}\right)$.

Theorem 9.1. The quotient $\widehat{G}^{\prime} / M^{\prime}$ represents the Picard functor $\mathrm{Pic}_{G / K}^{\tau}$ of translation invariant line bundles on $G$. The Poincaré bundle $P_{G \times G^{\prime}}$ on $G \times G^{\prime}$ is given by the triple $\left(\left(q \times q^{\prime}\right)^{*} P_{B \times B^{\prime}}, \Lambda, R\right)$ where

$$
\begin{aligned}
& \Lambda: M \times M^{\prime} \longrightarrow M^{\prime} \times M,\left(m, m^{\prime}\right) \longmapsto\left(m^{\prime}, m\right) \\
& R\left(m, m^{\prime}\right)=\left\langle m, m^{\prime}\right\rangle
\end{aligned}
$$

In terms of $\left(M \times M^{\prime}\right)$-linearization it is given by the mappings

$$
\begin{aligned}
\pi_{\left(m, m^{\prime}\right)}: P_{B \times B^{\prime}} & \longrightarrow P_{B \times B^{\prime}} \\
\omega & \longmapsto\left[\left\langle z, m^{\prime}\right\rangle \cdot\left\langle m, m^{\prime}\right\rangle\right] \otimes\left[\left\langle m, z^{\prime}\right\rangle \cdot \omega\right]
\end{aligned}
$$

where $z \in \widehat{G}, z^{\prime} \in \widehat{G}^{\prime}$ and $\omega$ is a point in the fiber $P_{q(z) \times q^{\prime}\left(z^{\prime}\right)}$. 
Here "." resp. " $\otimes "$ denotes the group law on $P_{B \times B^{\prime}}$ when $B$ resp. $B^{\prime}$ is regarded as a parameter space.

Ampleness of a line bundle on $G$ can also be described in terms of linearizations. The Poincaré bundle is a formal line bundle on $B \times B^{\prime}$, so it admits a trivialization such that the transition functions have constant absolute value, say of norm 1 . Therefore one obtains a well-defined absolute value on the total space $P_{B \times B^{\prime}}$.

Theorem 9.2. Let $L$ be a line bundle on $G$ and let $(N, \lambda, r)$ be the corresponding $M$-linearization. Then the following conditions are equivalent

(i) L is ample.

(ii) (a) $N$ is ample.

(b) $\langle\cdot, \lambda(\cdot)\rangle$ is positive definite on $M \times M$.

The condition (ii)(b) ensures that there are enough sections on $q^{*} N$.

Corollary 9.3. If $G_{K}$ is an abelian variety, then $B$ is an abelian scheme over $R$ and $\widehat{G}$ is a semi-abelian group scheme over $R$.

Mappings between Raynaud extensions $\Lambda: \widehat{G}_{1} / M_{1} \rightarrow \widehat{G}_{2} / M_{2}$ are in oneto-one correspondence with couples $(\lambda, \varphi)$ consisting of a group homomorphism $\lambda: M_{2}^{\prime} \rightarrow M_{1}^{\prime}$ and a morphism $\varphi: B_{1} \rightarrow B_{2}$ of formal abelian schemes such that the diagram

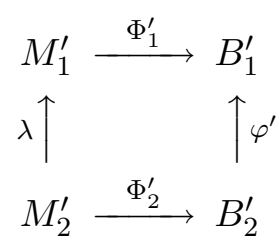

is commutative where $\varphi^{\prime}: B_{2}^{\prime} \rightarrow B_{1}^{\prime}$ is the dual of $\varphi$. Using this correspondence one obtains the following definition of polarizations.

Definition 9.4. A polarization of a pair $(\widehat{G}, M)$ consists of the following data:

(1) $\lambda_{M}: M \rightarrow M^{\prime}$ group homomorphism

(2) $\lambda_{B}: B \rightarrow B^{\prime}$ polarization of abelian schemes

such that the following conditions are satisfied:

(a) $\Phi^{\prime} \circ \lambda_{M}=\lambda_{B} \circ \Phi$

(b) $\left\langle m_{1}, \lambda_{M}\left(m_{2}\right)\right\rangle=\xi\left(\left\langle m_{2}, \lambda_{M}\left(m_{1}\right)\right\rangle\right)$ for all $m_{1} \in M_{1}, m_{2} \in M_{2}$.

(c) $\left\langle\cdot, \lambda_{M}(\cdot)\right\rangle$ is positive definite on $M \times M$.

Here $\xi$ denotes the symmetry on $\left(\operatorname{id}_{B} \times \lambda_{B}\right)^{*} P_{B \times B^{\prime}}$. 
Finally one shows:

Theorem 9.5. There is a one-to-one correspondence between the set of polarizations $\lambda_{G}$ of $G=\widehat{G} / M$ and the set of polarizations $\left(\lambda_{M}, \lambda_{B}\right)$ of $(\widehat{G}, M)$.

Now we will turn to the representability of the Picard functor of a proper smooth rigid variety which was settled by Hartl and the author in [22]. In the following we assume that $K$ is a local field with a discrete valuation.

Let $X_{K}$ be a smooth rigid space over $K$ which is proper and connected and assume that there exists a $K$-rational point $x: \operatorname{Sp} K \rightarrow X_{K}$ of $X_{K}$. Consider the Picard functor

$$
\underline{\mathrm{Pic}}_{X_{K} / K}:(\text { Smooth Rigid Spaces }) \longrightarrow(\text { Sets }), V_{K} \mapsto \underline{\mathrm{Pic}}_{X_{K} / K}\left(V_{K}\right)
$$

where

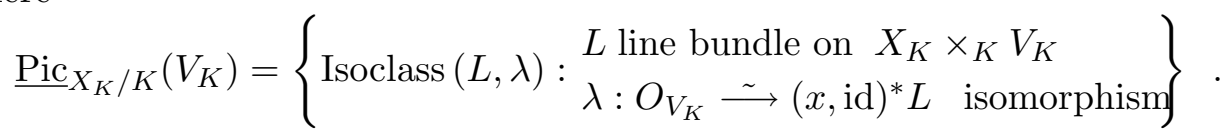

This is a contravariant functor. The main result is the representability of this functor under the additional assumption that $X_{K}$ admits a strict semi-stable formal model $X$ over the valuation ring $R$ associated to $K$.

Theorem 9.6. Under the conditions stated above, there exists a unique (up to canonical isomorphism) smooth rigid-analytic variety $\mathrm{Pic}_{X_{K} / K}$ and a natural transformation

$$
\Theta: \underline{\mathrm{Pic}}_{X_{K} / K} \longrightarrow \operatorname{Hom}_{K}\left({ }_{-}, \mathrm{Pic}_{X_{K} / K}\right)
$$

which is universal in the following sense: For any smooth rigid space $V_{K}$, the map

$$
\Theta\left(V_{K}\right): \underline{\operatorname{Pic}}_{X_{K} / K}\left(V_{K}\right) \stackrel{\sim}{\longrightarrow} \operatorname{Hom}_{K}\left(V_{K}, \operatorname{Pic}_{X_{K} / K}\right)
$$

is bijective.

In particular, there exists a line bundle $P$ on $X_{K} \times \mathrm{Pic}_{X_{K} / K}$ and an isomorphism $\lambda_{P}: O_{\mathrm{Pic}_{X_{K} / K}} \stackrel{\sim}{\longrightarrow}(x, \mathrm{id})^{*} P$ such that, for any smooth rigid space $V_{K}$ and for any pair $(L, \lambda) \in \underline{\mathrm{Pic}}_{X_{K} / K}\left(V_{K}\right)$, there is a unique morphism $\varphi: V_{K} \longrightarrow \operatorname{Pic}_{X_{K} / K}$ and a unique isomorphism $(L, \lambda) \stackrel{\sim}{\longrightarrow}(\mathrm{id} \times \varphi)^{*}\left(P, \lambda_{P}\right)$.

The identity component $\mathrm{Pic}_{X_{K} / K}^{0}$ of $\mathrm{Pic}_{X_{K} / K}$ is called the Picard variety of $X_{K}$ and $P$ is called the Poincaré bundle. The Picard variety is an extension of an abeloid variety by an affine torus. 
The Galois module $\operatorname{Pic}_{X_{K} / K}(\mathbb{K}) / \operatorname{Pic}_{X_{K} / K}^{0}(\mathbb{K})$ where $\mathbb{K}$ is the completion of the algebraic closure of $K$ is called the Néron-Severi group of $X_{K}$. The Néron-Severi group of $X_{K}$ is finitely generated.

The structure theorem of bounded rigid groups gave a hint how a Picard variety of a proper smooth rigid variety should look like. As we have seen in Theorem 8.10, there is a torus involved. Therefore one has to understand first the reason for the existence of such a torus. Due to the existence of a rig-étale cover which admits a semi-stable formal model 7.4, the source for the torus became clear by the following result:

Proposition 9.7. Assume that the residue field $k$ of $R$ is separably closed. Let $X_{K}$ be a smooth rigid analytic variety over $K$ which admits a strict semistable formal model $X$ over $\operatorname{Spf}(R)$. Let $R \rightarrow \tilde{R}$ be a finite extension of discrete valuation rings. Let $\tilde{V} \rightarrow \operatorname{Spf}(\tilde{R})$ be a strict semi-stable formal scheme. Assume that $\tilde{V}$ is small in the sense of $[22,1.4]$; so let $\tilde{V}$ be smooth over $\tilde{R}\left\langle\zeta_{1}, \ldots, \zeta_{t+1}\right\rangle /\left(\zeta_{1} \cdot \ldots \cdot \zeta_{t+1}-\tilde{\pi}\right)$. Let $\tilde{v} \in \tilde{V}(\tilde{R})$ be a point above $\left\{\zeta_{1}=\ldots=\zeta_{t}=\right.$ $1\}$. Consider a rigid analytic line bundle $\tilde{L}_{K}$ on $\left(X \times_{R} \tilde{V}\right)_{\text {rig }}$ which is trivialized along $\tilde{v}$.

Then $\tilde{L}_{K}$ is a tensor product $\tilde{L}_{K} \cong \tilde{M}_{K} \otimes \tilde{N}_{\text {rig }}$ of two line bundles, where

(1) $\tilde{M}_{K} \cong\left(\zeta_{1}^{n_{1}} \otimes \ldots \otimes \zeta_{t}^{n_{t}}\right)$ is the line bundle on $\left(X \times{ }_{R} \tilde{V}\right)_{\text {rig }}$ associated to suitable elements $n_{1}, \ldots, n_{t} \in \mathrm{H}^{1}\left(X_{0}, \mathbb{Z}\right)$. Such line bundles are called multiplicative.

(2) $\tilde{N}$ is a formal line bundle on $X \times_{R} \tilde{V}$.

The decomposition $\tilde{L}_{K} \cong \tilde{M}_{K} \otimes \tilde{N}_{\text {rig }}$ is unique. In particular, the Picard group of all line bundles which are trivial along $\tilde{v}$ decomposes into

$$
\operatorname{Pic}^{0}\left(\left(X \times_{R} \tilde{V}\right)_{\text {rig }}\right)=\mathrm{H}^{1}\left(X_{0}, \mathbb{Z}\right)^{t} \oplus \operatorname{Pic}^{0}\left(X \times_{R} \tilde{V}\right)
$$

Sketch of the proof of 9.6: One first shows the representability of $\mathrm{Pic}_{X / R}^{0}$ on the category of admissible reduced formal $R$-schemes by a smooth formal $R$-scheme $\bar{P}$ which is an extension of a formal torus $\bar{T}$ by an abelian formal $R$-scheme:

$$
\bar{T} \hookrightarrow \bar{P} \rightarrow B
$$


The interpretation of the torus is explained by the following diagram of canonical group homomorphisms

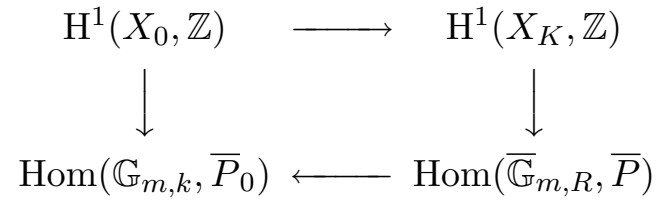

The vertical maps are given by sending a cocycle $n:=\left(n_{i j}\right)$ to the group homomorphism which sends a point $t$ of $\mathbb{G}_{m}$ to the line bundle given by the cocycle $\left(t^{n_{i j}}\right)$. The upper horizontal map is induced by the continuous reduction map $X_{K} \rightarrow X_{0}$. The lower horizontal map is given by reduction morphism. All these homomorphisms are bijective. In particular

$$
\mathrm{H}^{1}\left(X_{0}, \mathbb{Z}\right) \stackrel{\sim}{\longrightarrow} \mathrm{H}^{1}\left(X_{K}, \mathbb{Z}\right) \stackrel{\sim}{\longrightarrow} \operatorname{Hom}\left(\overline{\mathbb{G}}_{m, R}, \bar{T}\right) \stackrel{\sim}{\longrightarrow} \operatorname{Hom}\left(\overline{\mathbb{G}}_{m, k}, \bar{T}_{0}\right)
$$

are free of finite rank $r$ equal to the rank of the torus parts. The push-forward of the inclusion $\bar{T}_{\text {rig }} \hookrightarrow T_{K}$ of the torus of units into the affine torus yields a rigid analytic group $\widehat{P}_{K}$

$$
T_{K} \hookrightarrow \widehat{P}_{K} \rightarrow B_{\text {rig }} .
$$

The space $\widehat{P}_{K}$ represents the Picard functor of rigidified line bundles $L$ on the subcategory of smooth connected rigid spaces $\left(V_{K}, v\right)$ with rational base point such that $\mathrm{H}^{1}\left(V_{K}, \mathbb{Z}\right)=0$ and $L \mid\left(X_{K} \times_{V_{k}} v\right)$ is trivial. The true $\operatorname{Pic}_{X_{K} / K}^{0}$ is represented by a quotient of $\widehat{P}$ by the lattice $M_{K} \subset \widehat{P}_{K}$ consisting of the points corresponding to trivial line bundles. It can happen that the rank of $M_{K}$ is less than the rank of the torus $T_{K}$. Therefore the quotient

$$
P_{K}:=\widehat{P}_{K} / M_{K}
$$

representing $\operatorname{Pic}_{X_{K} / K}^{0}$ does not need to be proper. But it is an extension

$$
T_{K}^{a} \hookrightarrow P_{K} \rightarrow A_{K}
$$

of an abeloid variety $A_{K}$ by some affine torus $T_{K}^{a}$. There are examples, the $p$-adic Hopf surface [32], whose $\mathrm{Pic}^{0}$ is $\mathbb{G}_{m, K}$ and not proper. Nevertheless one can prove that the Néron-Severi group of $X_{K}$

$$
\mathrm{NS}_{X_{K} / K}:=\operatorname{Pic}_{X_{K} / K}(\bar{K}) / \operatorname{Pic}_{X_{K} / K}^{0}(\bar{K})
$$

is finitely generated. Here $\bar{K}$ denotes a complete algebraic closure of $K$. 


\section{REFERENCES}

[1] Artin, M.: Algebraic approximation over complete local rings, Publ. Math. IHES, 36, 23-58 (1969)

[2] Bosch, S.: A rigid analytic version of $M$. Artins's theorem on analytic equations, Math. Ann. 255, 395-404 (1981)

[3] Bosch, S.; Güntzer, U.; Remmert, R.: Non-Archimedean Analysis, Grundlehren der Mathematischen Wissenschaften, Bd. 261, Springer-Verlag, Berlin, Heidelberg, New York (1984)

[4] Bosch, S.; Lütkebohmert, W.: Stable Reduction and Uniformization of Abelian Varieties , Part I, Math. Ann. 270, 349-379 (1985) and Part II, Invent. Math. 78, 257-297 (1984)

[5] Bosch, S.; Lütkebohmert, W.: Degenerating abelian varieties, Topology 30, 653-698 (1991)

[6] Bosch, S.; Lütkebohmert, W.; Raynaud, M.: Formal and Rigid Geometry,

(1) Rigid Spaces, Math. Ann. 295, 291-317 (1993)

(2) Flattening Techniques, Math. Ann. 296, 403-429 (1993)

(3) The Relative Maximum Principle, Math. Ann. 302, 1-29 (1995)

(4) The Reduced Fiber Theorem, Invent. Math. 119, 361-398 (1995)

[7] Bosch, S.; Lütkebohmert, W.; Raynaud, M.: Néron Models, Springer, Ergeb. 3. Folge, Band 21, Springer-Verlag, Berlin, Heidelberg, New York (1990)

[8] Bosch, S.; Schlöter, K.: Néron models in the setting of formal and rigid geometry, Math. Ann. 301, 339-362 (1995).

[9] Deligne, P.; Rapoport, M.: Les schémas de modules de coubres elliptiques. Modular functions of one variable, II, Lecture Notes in Mathematics 349, Springer-Verlag, Berlin, Heidelberg, New York (1973)

[10] DE Jong, A.J.: Smoothness, Semi-stability and Alterations, Publ. Math. IHES 83, 51-93 (1996)

[11] ElkiK, R.: Solutions d'équations à coefficients dans un anneau hensélien, Ann. Scient. Ec. Norm. Sup., $4^{e}$ série, t. 6, 553-604 (1973)

[12] Faltings, G.; Chai, C.-L.: Degeneration of abelian varieties, Springer, Ergeb. 3. Folge, Band 22, Springer-Verlag, Berlin, Heidelberg, New York (1990)

[13] GerRitzen, L.: Zur nichtarchimedischen Uniformisierung von Kurven, Math. Ann. 210, 321-337 (1974)

[14] Gerritzen, L.; Grauert, H.: Die Azyklizität der affinoiden Überdeckugen. Global Analysis, Papers in Honor of K. Kodaira, 159-184. Tokyo: University of Tokyo Press, Princeton: Princeton University Press (1969)

[15] Goodman, J.; Hartshorne R.: Schemes with finite-dimensional cohomology groups, Am. J. Math. 91, 28-266 (1969)

[16] Grauert, H.; Remmert, R.: Über die Methode der diskret bewerteten Ringe in der nicht-archimedischen Analysis, Invent. math. 2, 87-133 (1966) 
[17] Grothendieck, A.; Dieudonné, J.: Eléments de Géométrie Algébrique, Publ. Math. IHES 4, 8, 11, 17, 20, 24, 28, 32 (1960-1967)

[18] Grothendieck, A.: Revêtements Étales et Groupe Fondamental; (SGA 1), Lecture Notes in Mathematics 224, Springer-Verlag, Berlin, Heidelberg, New York (1971)

[19] Grothendieck, A.: Schémas en Groupes I, II, III; (SGA 3), Lecture Notes in Mathematics 151, 52, 153, Springer-Verlag, Berlin, Heidelberg, New York (1970)

[20] Grothendieck, A.: Groupes de monodromie en géométrie algébriques; (SGA 7), Lecture Notes in Mathematics 288, 340, Springer-Verlag, Berlin, Heidelberg, New York (1972-73)

[21] HarTL, URS T.: Semi-stable models for rigid-analytic spaces, Manuscipta math. 110, 365-380 (2003)

[22] Hartl, Urs T.; Lütkebohmert, W.: On rigid-analytic Picard varieties for rigidanalytic spaces, J. reine angew. Math 528, 101-148 (2000)

[23] KIEHL, R.: Der Endlichkeitssatz für eigentliche Abbildungen in der nichtarchimedischen Funktionentheorie, Invent. Math. 2, 119 - 214 (1967)

[24] KienL, R.: Theorem A und B in der nichtarchimedischen Funktionentheorie, Invent. Math. 2, 256 - 273 (1967)

[25] LütкевонмеRт, W.: Formal-algebraic and rigid-analytic geometry, Math. Ann. 286, 341-371 (1990)

[26] LütкевонмеRт, W.: The structure of proper rigid groups, J. reine angew. Math. 468, 167-219 (1995)

[27] Mehlmann, F.: Flache Homomorphismen affinoider Algebren, Schriftenr. Math. Inst. Univ. Münster, 2. Serie, Heft 19 (1981)

[28] Martin, A.: Eine rigid-analytische Version des Artinschen Glättungssatzes, Schriftenr. Math. Inst. Univ. Münster, 3. Serie, Heft 33 (2008)

[29] Morikawa, H.: On theta functions and abelian varieties over valuation fields of rank one, Nagoya Journal 20, 1-27 (1962) and 21, 231-250 (1962)

[30] Mumford, D.: An analytic construction of degenerating curves over complete local rings, Comp. Math. 24, 129-174 (1972)

[31] Mumford, D.: An analytic construction of degenerating abelian varieties over complete rings, Comp.. Math. 24, 239-272 (1972)

[32] Mustafin, G.A.: p-adic Hopf varieties, Functional Analysis and its Applications 11, 234 - 235 (1977)

[33] NÉron, A.: Modèles minimeux des variétés abéliennes sur les corps locaux et globaux, Publ. Math. IHES, 21 (1964)

[34] Raynaud, M.: Variétés abéliennes et géométrie rigide, Actes du congrés international de Nice 1970, tome 1, p. 473-477.

[35] Raynaud, M.; Gruson, L.: Critères de platitude et de projectivité, Invent. Math. 13, 1-89 (1971)

[36] Raynaud, M.: Géométrie analytique rigide d'aprés Tate, Kiehl,..., Mémoires de la Société Mathématique de France 39-40, 319-327 (1974)

[37] Roquette, P.: Analytic theory of elliptic functions over local fields, Hamburger Math. Einzelschriften. Neue Folge, Heft 1 (1970) 
[38] TATE, J.: Rigid Analytic Spaces, Invent. Math. 12, 257-289 (1971)

[39] TATE, J.: A review of non-archimedean elliptic functions, Elliptic Curves, Modular Forma \& Fermat's Last Theorem. Series in Number Theory I, International press, 162-184 (1995)

[40] Temkin, M.: On local properties of non-archimedean analytic spaces, Math. Ann. 318, 585-607 (2000)

Werner Lütkebohmert

Dept. of Pure Mathematics

University of Ulm, 89069 Ulm, Germany

Email: Werner.Luetkebohmert@uni-ulm.de 